\title{
Oscillation modes in multi-resonant oscillator circuits
}

\author{
Franco Ramírez, Member, IEEE, Sergio Sancho, Member, IEEE, and Almudena Suárez, Fellow, IEEE
}

\begin{abstract}
An in-depth analysis of the oscillation modes in freerunning oscillators loaded with multi-resonance networks is presented. The analysis illustrates the mechanisms for the generation and stabilization of the various periodic modes and establishes the conditions for existence of a single stable periodic mode in distinct regions of the parameter plane. The mechanisms for the generation and stabilization of quasi-periodic regimes, with two concurrent oscillations, are also analyzed, considering different situations in terms of two relevant poles. The stability analysis of quasi-periodic solutions, derived in terms of admittance functions, can be applied to circuits simulated with harmonic balance, under the assumption of high quality factor resonators. The impact of the transistor biasing on the stability properties of the quasi-periodic regimes has been analyzed, demonstrating that it can be used to isolate the quasi-periodic solution from the periodic ones. The analysis procedures have been applied to a practical oscillator based on two cross-coupled transistors at the two frequencies $900 \mathrm{MHz}$ and $2.5 \mathrm{GHz}$. The case of two independent oscillations operating in a synchronized regime is also analysed, as well as its impact on the phase-noise behavior.
\end{abstract}

Index Terms-Multimode oscillation, stability, bifurcation, phase noise, dual oscillators

\section{INTRODUCTION}

$S_{\mathrm{a}}^{\mathrm{e}}$ everal works have been devoted to the in-depth bifurcation analysis of microwave circuits, such as frequency dividers, free-running and injection-locked oscillators and power amplifiers, among other [1]-[4]. However, few efforts have been made to address bifurcation analyses of multi-mode freerunning oscillators [5]-[9] and those are mostly limited to circuits having a symmetrical topology. In this symmetric case, the modes are a result of repeated eigenvalues [5]-[6] in the matrix describing either the linearized active network [9] or the passive network [5]-[7], which leads to different phase distributions at equivalent circuit nodes. In more general cases, the multiple oscillation modes are due to the loading of the active device(s), exhibiting broadband or multi-band negative resistance, with a multi-resonance network [10]-[19]. The multiple resonances are susceptible to give rise to multiple periodic and quasi-periodic oscillation modes, which can be unwanted, as in the case of crystal oscillators [10], or desired,

Manuscript received July 1, 2016. This work has been funded by the Spanish Government under contract TEC2014-60283-C3-1-R, the European Regional Development Fund (ERDF/FEDER) and the Parliament of Cantabria (12.JP02.64069). This paper is an expanded version from the 2016 IEEE MTTS International Microwave Symposium, San Francisco, CA, May 22-27, 2016 as in the case of dual-frequency oscillators [11]-[19], either concurrent or not. For instance, the interesting work [12] provides a valid prototype for a dual-frequency oscillator, based on a double-resonance load network (two parallel resonators in a series connection), in which the frequency is switched by changing the initial conditions. In [18]-[19] a similar topology is used to implement a concurrent dual-frequency oscillator for the simultaneous generation of two incommensurable frequencies, which should be of interest to reduce the power consumption in multi-band communication systems. Mathematical conditions for the physical existence of these quasi-periodic modes in particular circuit topologies have been provided in [13]. However, to the best of our knowledge, no previous work has presented an in-depth investigation of the mechanisms for the generation, stabilization and possible coexistence of the distinct periodic and quasi-periodic modes. This study should help resolve some of the uncertainties in the behavior of the multi-resonant oscillators, leading to well reported problems of jumps between different stable modes in crystal oscillators [10], for instance, or limiting their reliable application in multi-band systems. A significant difficulty is the autonomous nature of all the fundamental frequencies, which depend on the circuit parameters and increase the complexity of the stability analysis, especially in the case of quasi-periodic regimes, due to the existence of two or more Lyapunov exponents of zero value, as many as the number of incommensurable fundamental frequencies [20]-[22]. As in the case of frequency dividers or injection-locked oscillators (with operation bands delimited by bifurcations inherent to their operation [1]-[4]), one can expect some general properties in the behavior of multi-resonance oscillators, which would help derive criteria for a reliable performance.

The previous work [23] presented a detailed stability analysis of a free-running oscillator based on a cubic-nonlinearity model of the active device, loaded with a broadband multi-resonance network. Conditions were derived to ensure parameter regions in which only one particular periodic oscillation mode arises from the dc solution. This requires the existence of disjoint start-up regions in the parameter plane, delimited by their corresponding primary Hopf bifurcation loci [20]-[22],[24][25]. The investigation in [23] involved a stability analysis of the $\mathrm{dc}$ and periodic solutions, whereas and the stability

The authors are with the Communications Engineering Department, University of Cantabria, Santander, Spain. (suareza@unican.es; ramirezf@unican.es; sanchosm@unican.es). 
properties of the quasi-periodic solutions were conjectured with the aid of the Central Manifold Theorem [20]-[22]. These quasi-periodic solutions arise from secondary Hopf bifurcations of the periodic ones, and, according to [13], should be unstable in the case of active devices modeled with a cubic nonlinearity.

The work here extends the analytical investigation in [23] to cover cases in which the doubly autonomous quasi-periodic solutions can also be stable. This investigation is carried out in two stages. In a first stage, the active device is described with polynomial model as done, for instance, in [12], where the theoretical analysis is particularized to the double-resonance network. In the mathematical derivations presented here, there will be no restrictions regarding the load topology. On the other hand, the active device will be modeled with a fifth-order polynomial to enable the existence of physical quasi-periodic modes [15]. With this model, a thorough analytical investigation of the circuit behavior will be possible, without the use of numerical continuation methods. In this way it will be possible to perform an exhaustive detection of all the coexistent free-running modes of both periodic and quasiperiodic type. Stability and bifurcation analyses of the various kinds of solutions, in dc, periodic and quasi-periodic regime, will be carried out. Special attention will be paid to the possible coexistence of stable modes of the same or different nature. Additionally, the possible synchronization of the individual oscillations will be investigated, and the impact of this synchronization on the phase-noise behavior will be analyzed.

In a second stage, and departing from the comprehension of the global circuit behavior enabled by the analytical study, a methodology will be provided for the simulation of multiresonance oscillators in harmonic-balance (HB), using auxiliary generators (AGs) [24]-[25]. In view of the difficulties of the circuit-level stability analysis of doubly-autonomous quasiperiodic solutions, a semi-analytical approach, compatible with commercial HB, will also be presented. The analysis methods will be applied to a practical transistor-based oscillator, loaded with a double-resonant network, similar to the ones used in [12], [18]-[19].

The paper is organized as follows. Section II presents the steady-state, stability and bifurcation analysis of periodic modes. Section III addresses the stability analysis of the doubly-autonomous quasi-periodic modes. Section IV presents the application of a practical multi-mode oscillator, based on two cross-coupled transistors. Section V is devoted to the study of synchronized dual-mode concurrent oscillations and the phase-noise analysis.

\section{The Multiple Periodic Modes}

The study will be carried out considering an admittance-type model of the non-linear device(s). In fact, all the following equations and conditions, derived in terms of admittance functions, are susceptible to be used in combination with HB, through semi-analytical procedures, as will be shown in Section IV. In this section, and to get analytical insight into the circuit behavior, the active device will be modeled with a voltagecontrolled current source $i(v)$, as done in [12]. The use of a polynomial model will allow us to perform an exhaustive calculation of the coexistent oscillation modes, which would be virtually impossible with more complete models of the active devices, requiring error minimization techniques, sensitive to the initial values. The nonlinear device will be loaded with a general multi-resonance network, represented with the linear admittance $Y_{L}(j \omega)$.

The approximate analytical study will be carried out at the fundamental frequency only, under the assumption of sufficiently high quality factors in the resonators of $Y_{L}(j \omega)$. The describing function [26] associated with $i(v)$, is represented as $Y_{N}(V)$, where $V$ is the amplitude of the sinusoidal input voltage. Here the analysis in [23] is generalized to the case of a fifth-order voltage-controlled current source, which will be expressed as: $i(v)=a v+b v^{3}+d v^{5}$. This polynomial model with only odd terms enables a good estimation of the odd-mode behavior of cross-coupled transistors [27]-[28]. According to [13],[15], the introduction of fifth-order term should give rise to crucial changes in the circuit qualitative behavior, in comparison with [23]. The corresponding sinusoidal-input describing function is given by:

$$
Y_{N}(V)=a+\frac{3}{4} b V^{2}+\frac{5}{8} d V^{4}
$$

The coefficient $d$ has to be positive to suitably model the physical vanishing of negative conductance from certain voltage-excitation amplitude. Regarding the other two coefficients, there can be several possibilities. For $a<0$, the voltage-controlled current source will exhibit negative conductance in small signal. For $a>0, b<0$ and $d>0$, the device(s) will exhibit a negative conductance only under a large signal excitation. In the latter case, the oscillations will coexist with a stable dc regime. It is relevant to note that a same active device may exhibit $a<0, b>0$ and $d>0$ when biased above conduction threshold and $a>0, b<0$ and $d>0$ when biased below this threshold, so one can change the circuit qualitative behavior by modifying its bias point.

\section{A. Detection of periodic oscillation modes}

The stability of the dc solution is analyzed considering a small perturbation of complex frequency $s$ and linearizing the nonlinear device(s) about this dc solution, which provides the characteristic equation:

$$
Y_{N}(V \cong 0, s)+Y_{L}(s)=0
$$

where $Y_{N}(V \cong 0, s)$ is the function resulting from the linearization of the active device(s) about the particular bias point and $Y_{L}(s)$ is the linear admittance of the multi-resonance network, evaluated at the complex frequency s. Note that, unlike other previous works, such as [12]-[15], the formulation is based on a general representation of the linear load network, described with the admittance function $Y_{L}(s)$. Only in the application example, $Y_{L}(s)$ will be particularized to the doubleresonance network of Fig. 1, whereas in the previous work [23], $Y_{L}(s)$ was particularized to bandpass-type network. 
The poles of the dc solution are the roots of the characteristic equation (2). In the particular case of $i(v)=a v+b v^{3}+d v^{5}$, one obtains:

$$
\left.\frac{\partial i}{\partial v}\right|_{v=0}+Y_{L}(s)=a+Y_{L}(s)
$$

Because the load network in Fig. 1 contains four reactive elements, the dimension of the nonlinear system is $N=4$, so the characteristic equation (3) will provide four poles. Obviously, for $a>0$ all the roots of (3) will be on the left-hand-side of the complex plane (LHS). For $a<0$, and depending on the loadnetwork topology and element values, there can be one or more poles located on the right-hand-side (RHS). Distinct pairs of complex-conjugate poles crossing to the RHS will give rise to the generation of different periodic oscillation modes from the dc regime, each crossing corresponding to a primary Hopf bifurcation [23]. From the central manifold theorem [20]-[22], periodic modes generated from an unstable dc regime will be unstable in the neighborhood of the bifurcation.

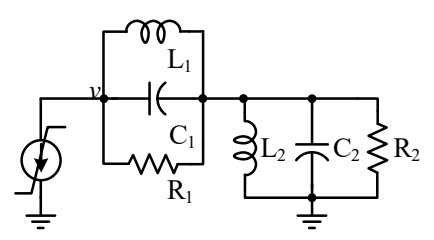

$\mathrm{i}(v)=\mathrm{a} v+\mathrm{b} v^{3}+\mathrm{d} v^{5}$

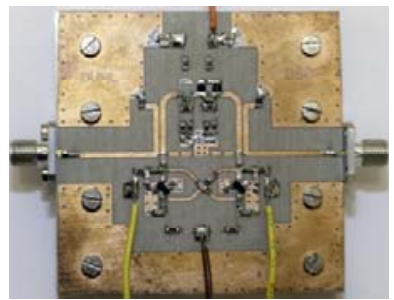

(b)

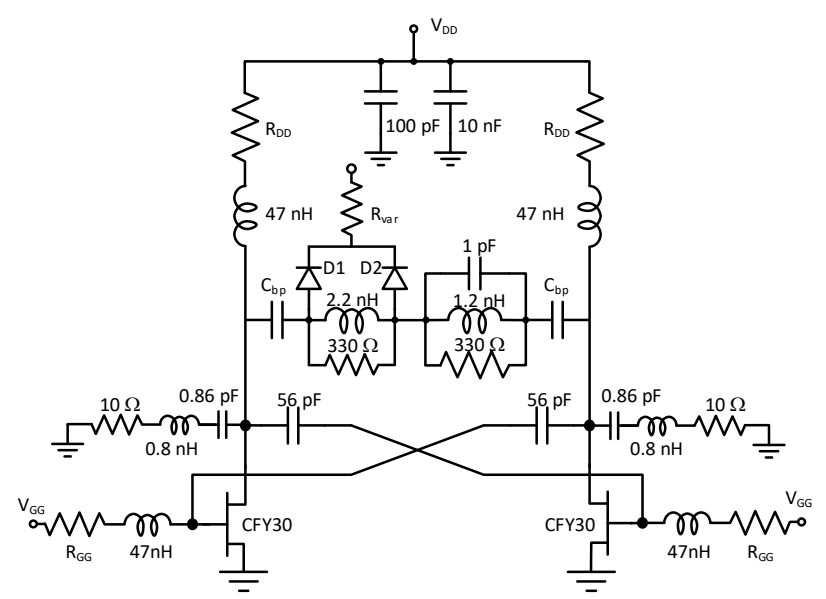

(c)

Fig. 1. Oscillator circuit loaded with a double-resonance network. (a) Active device(s) described with a polynomial model, for the analytical study. (b) and (c) Practical oscillator based on two cross-coupled transistors.

To avoid a repetition of contents, this section will focus on the behavior obtained for $a>0, b<0$ and $d>0$, which complements the investigation in [23]. In this case, the dc solution will always be stable. Despite this, periodic oscillations can exist, due to the coefficient $b<0$. In a practical oscillator, their onset from dc regime would take place for biasing conditions or element values different from the ones considered in the analysis. An example of an oscillator without a start-up from dc regime versus the analysis parameter can be found in [29].

Periodic oscillation modes will exist if the following steadystate oscillation condition is fulfilled:

$$
\begin{aligned}
& Y_{T}\left(V_{m}, \omega_{m}\right)=Y_{N}\left(V_{m}, j \omega_{m}\right)+Y_{L}\left(j \omega_{m}\right)=0 \\
& a+\frac{3}{4} b V_{m}^{2}+\frac{5}{8} d V_{m}^{4}+Y_{L}\left(j \omega_{m}\right)=0
\end{aligned}
$$

The subindex $m$ is used to emphasize the fact that there can be several distinct modes, with $m=1$ to $M$, with the frequency $\omega_{m}$ varying continuously versus the parameter. After the equal sign, the general admittance-type equation has been particularized to the polynomial model. For each root $\omega_{m}$ of the imaginary part $\operatorname{Im}\left[Y_{L}\left(j \omega_{m}\right)\right]=0$, the real part $\operatorname{Re}\left[Y_{L}\left(j \omega_{m}\right)\right]$ is computed, obtaining the amplitudes $V_{m}$ that fulfil $\operatorname{Re}\left[Y_{T}\left(V, \omega_{m}\right)\right]=0$.

For illustration, the commonly used load network [12]-[17], based on the series connection of two parallel resonators (Fig. $1)$, will be studied. The circuit element values considered are: $a=0 \Omega^{-1}, \quad b=-0.044 \Omega^{-1} / \mathrm{V}^{2}, \quad d=0.01 \Omega^{-1} / \mathrm{V}^{4}, \quad C_{1}=1 \mathrm{nF}$, $C_{2}=100 \mathrm{pF}, \quad f_{d}=907 \mathrm{MHz}, \quad f_{h}=3.903 \mathrm{GHz}, \quad R_{1}=25 \Omega$, $R_{2}=24.44 \Omega$, where $f_{d}$ and $f_{h}$ are the individual resonant frequencies of the two resonators, with the respective quality factors $Q_{d}=126$ and $Q_{h}=60$. The use of $a \neq 0$ would complicate the solution curves without adding any relevant information, since, as will be shown, in the presence of $b<0$, the periodic curves will necessarily exhibit turning points, regardless of $a$. The circuit will be analyzed versus the activedevice parameter $d$. Initially, the resistor value $R_{1}=30 \Omega$ will be considered. This provides the solution diagram in Fig. 2, where the amplitude $V_{m}$ of each oscillation has been traced versus $d$. The analysis shows two distinct periodic oscillation modes, represented in solid line, one at the lower resonant frequency (about $f_{d}=907 \mathrm{MHz}$ ) and the other at the higher resonant frequency (about $f_{h}=3.903 \mathrm{GHz}$ ). Each periodic solution curve exhibits a turning point (or infinite-slope point). This turning point is $\mathrm{T}_{1}\left(\mathrm{~T}_{2}\right)$ for the solution curve at $f_{d}\left(f_{h}\right)$ [see Fig. 2]. Therefore, two different oscillatory solutions exist for each mode. This is due to the signs of the active-device coefficients $(b<0, d>0)$, which give rise to an increase and then a reduction of the negative conductance with the excitation amplitude, so two different amplitudes fulfill (4) for each oscillation mode.

\section{B. Stability analysis of the periodic oscillation modes}

The stability analysis of each periodic solution is performed by considering a small amplitude perturbation of complex frequency s. To obtain the characteristic system, the active device is linearized about the corresponding periodic solution and the linear network is evaluated at $-j \omega_{m}+s, s, j \omega_{m}+s$ [24]-[25],[30]-[31]. For a better insight, the analysis will be particularized to the nonlinear characteristic $i(v)$. Obtaining the Jacobian matrix associated with $i(v)$ involves a previous 
calculation of the derivative $g(v)=\partial i / \partial v[1]-[2]$, expressed in a Fourier series as $g(v)=\sum_{k} G_{k} e^{j k \omega_{m} t}$. When limiting the analysis to the fundamental frequency, this Fourier expansion should go from $k=-2$ to $k=2$. The characteristic system is:

$$
\left\{\left[\begin{array}{ccc}
G_{m, 0} & G_{m,-1} & G_{m,-2} \\
G_{m, 1} & G_{m, 0} & G_{m,-1} \\
G_{m, 2} & G_{m, 1} & G_{m, 0}
\end{array}\right]+\left[\begin{array}{ccc}
Y_{L}(-j \omega+s) & 0 & 0 \\
0 & Y_{L}(s) & 0 \\
0 & 0 & Y_{L}(j \omega+s)
\end{array}\right]\right\}\left[\begin{array}{c}
\Delta V_{-1} \\
\Delta V_{0} \\
\Delta V_{1}
\end{array}\right]=0 \text { (5) }
$$

where the subindex $m$ indicates the particular oscillation mode.

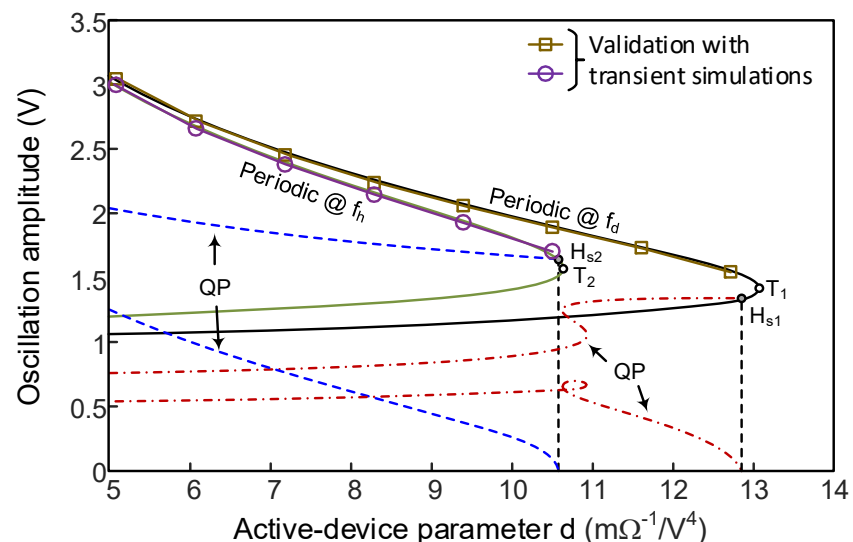

Fig. 2. Solutions of the circuit in Fig. 1(a) traced versus the active-device parameter $d$ for $R_{1}=30 \mathrm{Ohm}$ in the lower-frequency resonator. The two components (at $f_{d}$ and $f_{h}$ ) of the quasi-periodic solution generated at $\mathrm{H}_{\mathrm{s} 1}\left(\mathrm{H}_{\mathrm{s} 2}\right)$ are traced in dash-dotted (dashed) line. Solution points obtained with timedomain integration are superimposed (square and circle markers).

The poles are given by the roots of the determinant of the characteristic matrix [between the braces in (5)]. In the case of $\partial \mathrm{i} / \partial v=a+3 b v^{2}+5 d v^{4}$, the matrix on the left hand side of (5), renamed as $\left[G_{m}\right]$, is given by:

$\left[G_{m}\right]=\left[\begin{array}{ccc}a+6 b V_{m, 1} V_{m,-1}+ & 0 & 3 b V_{m,-1}{ }^{2}+ \\ 30 d V_{m, 1}{ }^{2} V_{m,-1}{ }^{2} & & 20 d V_{m,-1}{ }^{3} V_{m, 1} \\ 0 & a+6 b V_{m, 1} V_{m,-1}+ & 0 \\ 3 b V_{m, 1}{ }^{2}+ & 30 d V_{m, 1}{ }^{2} V_{m,-1}{ }^{2} & \\ 20 d V_{m, 1}{ }^{3} V_{m,-1} & 0 & a+6 b V_{m, 1} V_{m,-1}+ \\ & & 30 d V_{m, 1}{ }^{2} V_{m,-1}{ }^{2}\end{array}\right]$

where $V_{m, 1}=V_{m,-1}=V_{m} / 2$. For a sufficiently high quality factor, $G_{m, 2}$ and $G_{m,-2}$ can be neglected, so (5) becomes the complex equation:

$$
G_{m, o}\left(V_{m, 1}, V_{m,-1}\right)+Y_{L}(s)=0
$$

which is formally identical to the one that would be obtained using the incremental describing function [27]. This simpler equation will be useful to get analytical insight into the system behavior. When particularized to the polynomial model, one obtains:

$$
\begin{aligned}
G_{m, o}+Y_{L}(s) & =a+6 b V_{m, 1} V_{m,-1}+30 d V_{m, 1}{ }^{2} V_{m,-1}^{2}+Y_{L}(s) \\
& =a+3 b \frac{V_{m}^{2}}{2}+15 d \frac{V_{m}^{4}}{8}+Y_{L}(s)=0
\end{aligned}
$$

Because the load network in Fig. 1 contains four reactive elements, the above characteristic equation will provide four poles, two of them being complex-conjugate poles at the oscillation frequency $\omega_{m}$, equivalent to one real pole, due to the non-univocal relationship between Floquet exponents and multipliers [22],[32]. Application of this stability analysis to the solutions in Fig. 2 leads to the results in Fig. 3(a).

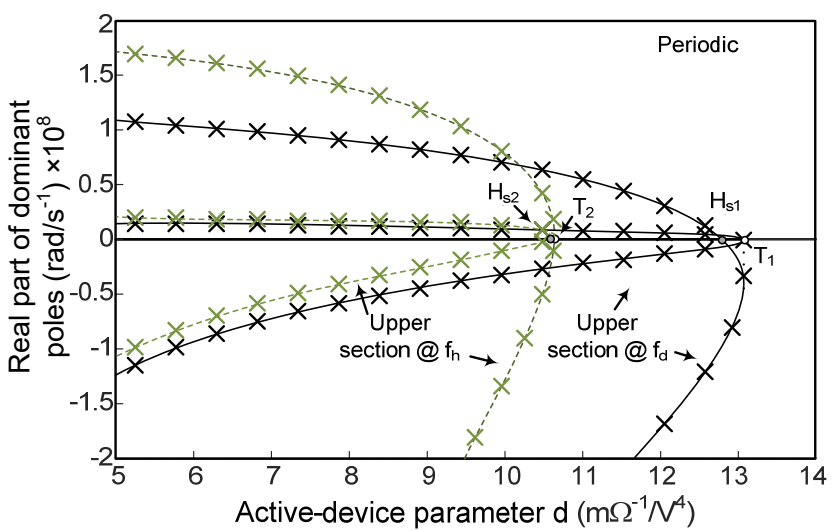

(a)

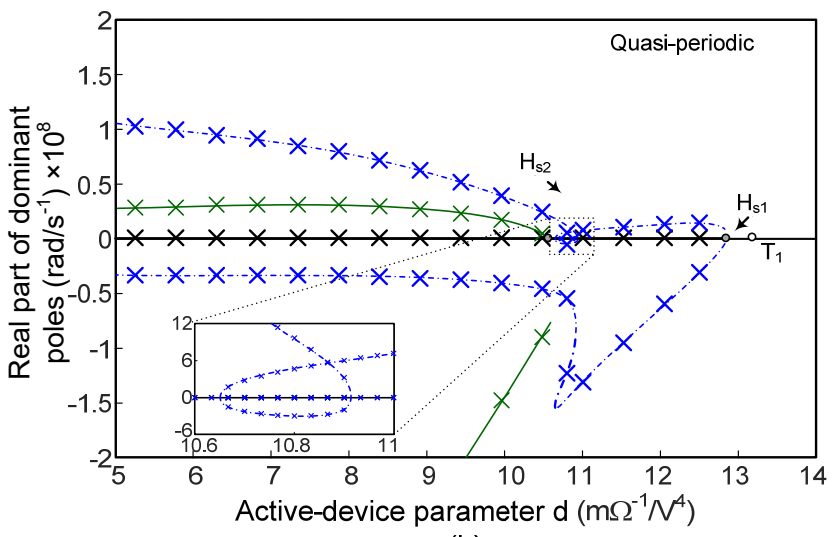

(b)

Fig. 3. Stability analysis of the steady-state solutions in the bifurcation diagram in Fig. 2. (a) Stability analysis of the periodic solutions. The real part of the poles has been traced versus the analysis parameter $d$. (b) Stability analysis of the quasi-periodic solutions.

The real part of the poles has been represented versus $d$. The oscillation mode at $f_{d}$ is stable up to the turning point $\mathrm{T}_{1}$, where a real pole crosses through zero to the RHS. Once in the lower section of the curve at $f_{d}$, a secondary Hopf bifurcation takes place at the point $\mathrm{H}_{\mathrm{s} 1}$, where a second fundamental frequency at $f_{h}$ is generated. For $d$ values smaller than the one corresponding 
to $\mathrm{H}_{\mathrm{s} 1}$, the lower section of the curve at $f_{d}$ is unstable, with three poles on the RHS. In the case of the periodic solution curve at $f_{h}$, the upper section is stable up to the secondary Hopf bifurcation $\mathrm{H}_{\mathrm{s} 2}$, where a pair of complex conjugate poles crosses to the RHS. In addition, a real pole crosses to the RHS at $\mathrm{T}_{2}$. Therefore, for $d<10.6 \mathrm{~m} \Omega^{-1} / \mathrm{V}^{4}$ the two periodic solution curves contain stable sections. Observation of one periodic mode or another will depend on the initial values. However, some sections of the quasi-periodic solutions in Fig. 2 could also be stable. The coexistence of periodic solutions predicted by the analyses in Fig. 2 and Fig. 3 has been validated with transient simulations, in commercial software. To obtain the coexistent stable solutions, initial values have been provided to the transient simulator in a systematic manner. A sweep has been carried out in the initial value of the device voltage $v$, in Fig. 1(a). This way it has been possible to obtain convergence to the distinct coexistent stable solutions, since reaching one or another depends on the initial conditions. Traces plotted with markers (squares and circles) in Fig. 2 indicate the results of this transient analysis.

\section{Bifurcation loci}

In the general case, to ensure disjoint parameter regions with a single stable periodic solution one should trace three different bifurcation loci [20-22,24-25] in the parameter plane. These are the primary Hopf locus, associated with the generation/extinction of a periodic oscillation from a $d c$ regime, the turning-point (TP) locus, composed of points at which the periodic solution curves exhibit an infinite slope, and the secondary-Hopf locus (SHL), associated with the generation/extinction of a quasi-periodic solution.

\section{a) Primary Hopf bifurcations}

In terms of two parameters $\eta_{1}, \eta_{2}$ (such as a device bias voltage or a varactor capacitance), the locus of primary Hopf bifurcations is given by:

$$
H_{p}\left(\eta_{1}, \eta_{2}\right)=Y_{N}\left(V_{m} \cong 0, j \omega_{m}\right)+Y_{L}\left(j \omega_{m}\right)=a+Y_{L}\left(j \omega_{m}\right)
$$

where the general equation has been particularized to $i(v)$ after the second equal sign. The subindex $m$ emphasizes the fact that there will be a different Hopf locus for each oscillation mode. The existence of these loci requires $a<0$. As shown in [23], for the different periodic modes to have a physical onset from the $\mathrm{dc}$ regime in distinct parameter intervals, the loci must give rise to disjoint regions in the parameter plane. This case will be considered in the analysis of the practical oscillator in Fig. 1(b), presented in Section IV.

\section{b) Secondary Hopf bifurcations}

At a secondary Hopf bifurcation, a pair of complex-conjugate poles of the periodic regime at $\omega_{m}$ take the purely imaginary values $\pm j \Omega$. The secondary Hopf locus $H_{s}$ is easily calculated by replacing $s$ with $\pm j \Omega$ in (8), taking into account that the steady-state condition (4) must also be fulfilled. This leads to the complex-equation system:

$$
\begin{aligned}
& H_{1, s}\left(\eta_{1}, \eta_{2}\right)=G_{0}\left(V_{m}\right)+Y_{L}(j \Omega)=0 \\
& a+3 b \frac{V_{m}^{2}}{2}+15 d \frac{V_{m}^{4}}{8}+Y_{L}(j \Omega)=0 \\
& H_{2, s}\left(\eta_{1}, \eta_{2}\right)=Y_{N}\left(V_{m}, j \omega_{m}\right)+Y_{L}\left(j \omega_{m}\right)=0 \\
& a+\frac{3}{4} b V_{m}^{2}+\frac{5}{8} d V_{m}^{4}+Y_{L}\left(j \omega_{m}\right)=0
\end{aligned}
$$

where the subindex $m$ indicates the oscillation mode, since different secondary Hopf-bifurcation loci may be obtained, each resulting from one of the distinct periodic oscillations. In the case of the circuit in Fig. 1(a), system (10) provides the two secondary Hopf bifurcation loci traced in solid line in Fig. 4, one corresponding to oscillations generated from the mode at $f_{d}$ (extending to higher $d$ values) and the other corresponding to oscillations generated from the mode at $f_{h}$. For $R_{1}=30 \Omega$, one obtains the two secondary-Hopf bifurcations detected in Fig. 2 and Fig. 3(a).

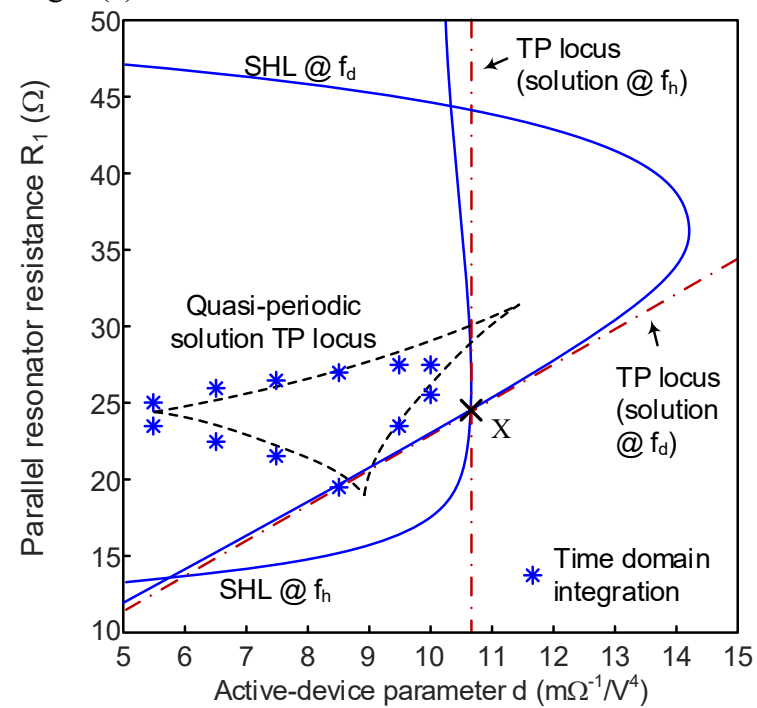

Fig. 4. Bifurcation loci of the circuit in Fig. 1(a), traced in the plane defined by the active-device parameter $d$ and the resistor $R_{1}$. SHL stands for "secondary Hopf locus".

\section{c) Turning points}

At a turning point, the periodic solution curve exhibits an infinite slope, so the Jacobian matrix associated with the complex steady-state equation (4) must be singular. For a circuit described in terms of the total admittance function $Y_{T}$, this condition is given by:

$$
\begin{gathered}
T_{p}\left(\eta_{1}, \eta_{2}\right)=\operatorname{det}\left[\begin{array}{ll}
\frac{\partial Y_{T}{ }^{r}}{\partial V_{m}} & \frac{\partial Y_{T}{ }^{r}}{\partial \omega_{m}} \\
\frac{\partial Y_{T}^{i}}{\partial V_{m}} & \frac{\partial Y_{T}{ }^{i}}{\partial \omega_{m}}
\end{array}\right]=\frac{\partial Y_{T}^{r}}{\partial V_{m}} \frac{\partial Y_{T}{ }^{i}}{\partial \omega_{m}}=0 \\
\left(\frac{10}{8} d V_{m}^{2}+\frac{3}{4} b\right) 2 V_{m} \frac{\partial \operatorname{Im}\left(Y_{L}\right)}{\partial \omega_{m}}=0
\end{gathered}
$$

where the superindexes $r$ and $i$ indicate real and imaginary parts, respectively. As in all the previous derivations, on the right side, the general condition has been particularized to the 
case of $i(v)$. With high quality factors, the more usual cause for the observation of a turning point will be $\partial Y_{T}^{r} / \partial V_{m}=0$. When particularizing to the polynomial model, the turning points will be obtained for the voltage amplitude:

$$
V_{P}=\sqrt{-\frac{3}{5} \frac{b}{d}}
$$

So turning points will only be possible for $b<0$ and $d>0$. The periodic-oscillation amplitude at the turning point does not depend on the particular oscillation mode. It is also independent of the device linear admittance $a$. Replacing the expression (12) into the steady-state oscillation condition, one obtains the turning-point locus in periodic regime $T_{p}\left(\eta_{1}, \eta_{2}\right)$ :

$$
T_{p}\left(\eta_{1}, \eta_{2}\right)=a+\frac{3}{4} b V_{P}^{2}+\frac{5}{8} d V_{P}^{4}+Y_{L}\left(j \omega_{m}\right)=0
$$

Assuming constant values of the device coefficients, the load network must fulfill $\operatorname{Re}\left(Y_{L 1}\right) \neq \operatorname{Re}\left(Y_{L 2}\right) \neq \ldots \neq \operatorname{Re}\left(Y_{L M}\right)$ to prevent the various oscillation modes from exhibiting a turning point (and becoming stable) at the same parameter values. Fig. 4 shows the turning-point loci corresponding to the two oscillation modes of the circuit of Fig. 1(a). As expected, the turning point in the $f_{h}$ mode is nearly independent of $R_{1}$ since this resistor belongs to the lower frequency resonator. The two turning point loci intersect at the point $X$. When decreasing $d$ from a high value, above (below) $X$, the periodic oscillation at $f_{d}\left(f_{h}\right)$ will be the only stable one between the turning point locus at $f_{d}\left(f_{h}\right)$ and the turning point locus at $f_{h}\left(f_{d}\right)$.

\section{Doubly-Autonomous Quasi-Periodic Regime}

The onset of doubly autonomous oscillation modes will be undesired in dual or multi-mode periodic oscillators, since they will limit the usable parameter intervals of these circuits. However, in concurrent dual-frequency oscillators [18]-[19], this will be the intended operation regime. In both cases, it will be interesting to investigate its stability properties and the conditions required for their stabilization under the variation of the circuit parameters.

\section{A. Calculation of the quasi-periodic oscillation modes}

The case of a doubly-autonomous quasi-periodic regime at the two fundamental frequencies $\omega_{m}$ and $\omega_{n}$ will be analyzed. For high quality factor resonators, it will be possible to neglect the intermodulation terms [15]-[16],[18]-[19]. To calculate the active-device admittance functions, the input voltage $v(t)=V_{1} \cos \left(\omega_{1} t\right)+V_{2} \cos \left(\omega_{2} t\right)$ is considered, where the subindexes 1,2 refer to the components at $\omega_{m}$ and $\omega_{n}$, for notation simplicity. The phase at each frequency component can be arbitrarily set to zero, since the two frequencies are incommensurable. Note that in the presence of a multiresonance load network there can be multiple combinations of $\omega_{m}$ and $\omega_{n}$, as shown in [23]. When introducing $v(t)$ into the nonlinear function $i(v)$ [13], one obtains:

$$
\begin{aligned}
& Y_{1}\left(V_{1}, V_{2}\right)=\frac{5}{8} d V_{1}^{4}+\frac{3}{4} b V_{1}^{2}+\frac{15}{4} d V_{1}^{2} V_{2}^{2}+\frac{3}{2} b V_{2}^{2}+\frac{15}{8} d V_{2}^{4}+a \\
& Y_{2}\left(V_{1}, V_{2}\right)=\frac{15}{8} d V_{1}^{4}+\frac{3}{2} b V_{1}^{2}+\frac{15}{4} d V_{1}^{2} V_{2}^{2}+\frac{3}{4} b V_{2}^{2}+\frac{5}{8} d V_{2}^{4}+a
\end{aligned}
$$

As expected, when particularizing $Y_{2}\left(V_{1}, V_{2}\right)$ to $V_{2}=0$ one obtains the incremental describing function $G_{0}$ that enables the stability analysis of the periodic oscillation mode at $\omega_{1}$ [see (8) ]. The steady-state doubly-autonomous quasi-periodic solutions are calculated by solving:

$$
\begin{gathered}
Y_{T 1}\left(V_{1}, V_{2}, \omega_{1}\right)=Y_{1}\left(V_{1}, V_{2}\right)+Y_{L}\left(j \omega_{1}\right)=0 \\
Y_{T 2}\left(V_{1}, V_{2}, \omega_{2}\right)=Y_{2}\left(V_{1}, V_{2}\right)+Y_{L}\left(j \omega_{2}\right)=0
\end{gathered}
$$

The solutions of (15) have been added to Fig. 2. As expected, there are two different quasi-periodic curves, arising from the mode at $f_{d}\left(f_{h}\right)$, from zero amplitude at the second fundamental frequency $f_{h}\left(f_{d}\right)$ at the secondary Hopf bifurcation $\mathrm{H}_{\mathrm{s} 1}\left(\mathrm{H}_{\mathrm{s} 2}\right)$. The two components (at $f_{d}$ and $f_{h}$ ) of the quasi-periodic solution generated at $\mathrm{H}_{\mathrm{s} 1}\left(\mathrm{H}_{\mathrm{s} 2}\right)$ are traced in dash-dotted (dashed) line.

\section{B. Stability analysis of the doubly-autonomous quasi- periodic solutions}

The stability analysis of the doubly-autonomous quasi-periodic regime requires a small perturbation of the two-tone steadystate solution, which, in the case of an active device $i(v)$, leads to the following characteristic equation:

$$
\left\{[M C]+\left[Y_{L}\left(j \omega_{1}, j \omega_{2}, s\right)\right]\right\} \Delta \bar{X}=0
$$

where $\Delta \bar{X}$ contains all the perturbation components $\Delta X_{k, l}$ of the node voltage, of complex nature, where the subindexes indicate intermodulation terms of the form $k \omega_{1}+l \omega_{2}$. On the other hand, [MC] represents the conversion matrix [1]-[2] obtained by linearizing the active-device model $i(v)$ about the quasi-periodic solution. This matrix is composed of the terms:

$$
\begin{gathered}
{[M C]=\left(\frac{\partial I_{k, l}}{\partial X_{r, p}}\right)_{k, l, r, p}} \\
k, r=-Q, \ldots, Q, \\
l, p=-N, \ldots, N
\end{gathered}
$$

The current $I_{k, l}$ can be expressed as $I_{k, l}=Y_{k, l}(\bar{X}) X_{k, l}$. In the case considered here of incommensurable fundamental frequencies, it can be demonstrated that:

$$
\frac{\partial I_{k, l}}{\partial X_{r, p}}=G_{k-r, l-p}
$$

where $G_{p, q}$ is the intermodulation component at $p \omega_{1}+q \omega_{2}$ of the derivative $g(v)=\partial i / \partial v$. In the case $Q=N=1$, and avoiding the mixing terms in $I_{p, q}$ and $X_{p, q}$, under the assumption of high quality factors, one obtains:

$$
[M C]=\left[\begin{array}{ccccc}
G_{0,0} & G_{-1,1} & G_{-1,0} & G_{-1,-1} & G_{-2,0} \\
G_{1,-1} & G_{0,0} & G_{0,-1} & G_{0,-2} & G_{-1,-1} \\
G_{1,0} & G_{0,1} & G_{0,0} & G_{0,-1} & G_{-1,0} \\
G_{1,1} & G_{0,2} & G_{0,1} & G_{0,0} & G_{-1,1} \\
G_{2,2} & G_{1,1} & G_{1,0} & G_{1,-1} & G_{0,0}
\end{array}\right] \text { (19) }
$$


The above matrix is the quasi-periodic equivalent of the one in (5). In turn, the passive-network matrix $Y_{L}$ is given by:

$$
\begin{aligned}
& {\left[Y_{L}\left(j \omega_{1}, j \omega_{2}, s\right)\right]=} \\
& {\left[\begin{array}{ccccc}
Y_{L}\left(-j \omega_{1}+s\right) & 0 & 0 & 0 & 0 \\
0 & Y_{L}\left(-j \omega_{2}+s\right) & 0 & 0 & 0 \\
0 & 0 & Y_{L}(s) & 0 & 0 \\
0 & 0 & 0 & Y_{L}\left(j \omega_{1}+s\right) & 0 \\
0 & 0 & 0 & 0 & Y_{L}\left(j \omega_{2}+s\right)
\end{array}\right]}
\end{aligned}
$$

Rewriting (16) at the positive spectrum only, the characteristic system can be reduced to:

$$
\begin{aligned}
G_{1,1} \Delta X_{-2}+G_{0,2} \Delta X_{-1}+G_{0,0} \Delta X_{1} & +G_{-1,1} \Delta X_{2}+ \\
& +Y_{L}\left(j \omega_{1}+s\right) \Delta X_{1}=0, \\
G_{2,0} \Delta X_{-2}+G_{1,1} \Delta X_{-1}+G_{1,-1} \Delta X_{1} & +G_{0,0} \Delta X_{2}+ \\
& +Y_{L}\left(j \omega_{2}+s\right) \Delta X_{2}=0, \\
\Delta X_{ \pm 1} \equiv \Delta X_{ \pm 1,0}=\Delta V_{1} \pm j \Delta \phi_{1}, & \\
\Delta X_{ \pm 2} \equiv \Delta X_{0, \pm 1}=\Delta V_{2} \pm j \Delta \phi_{2} &
\end{aligned}
$$

where $\Delta V_{k}$ and $\Delta \phi_{k}$ are the amplitude and phase perturbation components. To get insight into the stability properties of doubly-autonomous quasi-periodic solutions, some aspects must be taken into account. The dimension of the nonlinear dynamical system governing the circuit behavior agrees with the number of its reactive elements. In the case of $i(v)$, loaded with the passive network in Fig. 1, this dimension is $N=4$. Therefore, the stability properties of each steady-state solution are defined by four Lyapunov exponents, which are real quantities [20]-[22]. In the case of a doubly-autonomous quasiperiodic solution, two of these Lyapunov exponents are equal to zero, so $\lambda_{3}=\lambda_{4}=0$.

In consistency with this, two poles of the characteristic system (19) will have a zero value. The stability properties will be defined by the two remaining Lyapunov exponents $\lambda_{1}, \lambda_{2}$, associated with two distinct poles of (21). These poles can be either real or complex conjugate. In the latter case, it is assumed that the poles have a small frequency value, which will be justified later in this section. Then, it is reasonable to consider a small value of $s$, so it will be possible to expand $Y_{L}$ in a firstorder Taylor series about the steady-state quasi-periodic regime. System (21) will be split into real and imaginary parts using $\Delta X_{-k}=\Delta X_{k}^{*}, G_{-k,-l}=G_{k, l}^{*}$. It will also be taken into account that $s$ acts like a time differentiator of the envelopes $\left[V_{1}+\Delta V_{1}(t)\right] e^{j \Delta \phi_{1}(t)},\left[V_{2}+\Delta V_{2}(t)\right] e^{j \Delta \phi_{2}(t)}$ affected by $Y_{L}$. This leads to:

$$
\begin{aligned}
& \frac{\partial Y_{T 1}^{r}}{\partial V_{1}} \Delta V_{1}+\frac{\partial Y_{T 1}^{r}}{\partial V_{2}} \Delta V_{2}+\frac{\partial Y_{T 1}^{i}}{\partial \omega_{1}} \frac{\Delta \dot{V}_{1}}{V_{1 o}}+\frac{\partial Y_{T 1}^{r}}{\partial \omega_{1}} \Delta \dot{\phi}_{1}=0 \\
& \frac{\partial Y_{T 1}^{i}}{\partial V_{1}} \Delta V_{1}+\frac{\partial Y_{T 1}^{i}}{\partial V_{2}} \Delta V_{2}-\frac{\partial Y_{T 1}^{r}}{\partial \omega_{1}} \frac{\Delta \dot{V}_{1}}{V_{1 o}}+\frac{\partial Y_{T 1}^{i}}{\partial \omega_{1}} \Delta \dot{\phi}_{1}=0 \\
& \frac{\partial Y_{T 2}^{r}}{\partial V_{1}} \Delta V_{1}+\frac{\partial Y_{T 2}^{r}}{\partial V_{2}} \Delta V_{2}+\frac{\partial Y_{T 2}^{i}}{\partial \omega_{2}} \frac{\Delta \dot{V}_{2}}{V_{2 o}}+\frac{\partial Y_{T 2}^{r}}{\partial \omega_{2}} \Delta \dot{\phi}_{2}=0 \\
& \frac{\partial Y_{T 2}^{i}}{\partial V_{1}} \Delta V_{1}+\frac{\partial Y_{T 2}^{i}}{\partial V_{2}} \Delta V_{2}-\frac{\partial Y_{T 2}^{r}}{\partial \omega_{2}} \frac{\Delta \dot{V}_{2}}{V_{2 o}}+\frac{\partial Y_{T 2}^{i}}{\partial \omega_{2}} \Delta \dot{\phi}_{2}=0
\end{aligned}
$$

where the superindexes $r$ and $i$ stand for the real and imaginary parts. Solving for the vector of time derivatives in (22) provides:

$$
\left[\begin{array}{c}
\frac{\Delta \dot{V}_{1}}{V_{1 o}} \\
\frac{\Delta \dot{V}_{2}}{V_{2 o}} \\
\Delta \dot{\phi}_{1} \\
\Delta \dot{\phi}_{2}
\end{array}\right]=\left[\begin{array}{cccc}
a_{11} & a_{12} & 0 & 0 \\
a_{21} & a_{22} & 0 & 0 \\
0 & 0 & 0 & 0 \\
0 & 0 & 0 & 0
\end{array}\right]\left[\begin{array}{l}
\Delta V_{1} \\
\Delta V_{2} \\
\Delta \phi_{1} \\
\Delta \phi_{2}
\end{array}\right](23)
$$

where:

$$
\begin{aligned}
& a_{11}=-\left(\frac{\partial Y_{T 1}^{r}}{\partial V_{1}} \frac{\partial Y_{T 1}^{i}}{\partial \omega_{1}}-\frac{\partial Y_{T 1}^{r}}{\partial \omega_{1}} \frac{\partial Y_{T 1}^{i}}{\partial V_{1}}\right) /\left|\frac{\partial Y_{T 1}}{\partial \omega_{1}}\right|^{2} \\
& a_{12}=-\left(\frac{\partial Y_{T 1}^{r}}{\partial V_{2}} \frac{\partial Y_{T 1}^{i}}{\partial \omega_{1}}-\frac{\partial Y_{T 1}^{r}}{\partial \omega_{1}} \frac{\partial Y_{T 1}^{i}}{\partial V_{2}}\right) /\left|\frac{\partial Y_{T 1}}{\partial \omega_{1}}\right|^{2} \\
& a_{21}=-\left(\frac{\partial Y_{T 2}^{r}}{\partial V_{1}} \frac{\partial Y_{T 2}^{i}}{\partial \omega_{2}}-\frac{\partial Y_{T 2}^{r}}{\partial \omega_{2}} \frac{\partial Y_{T 2}^{i}}{\partial V_{1}}\right) /\left|\frac{\partial Y_{T 1}}{\partial \omega_{2}}\right|^{2} \\
& a_{22}=-\left(\frac{\partial Y_{T 2}^{r}}{\partial V_{2}} \frac{\partial Y_{T 2}^{i}}{\partial \omega_{2}}-\frac{\partial Y_{T 2}^{r}}{\partial \omega_{2}} \frac{\partial Y_{T 2}^{i}}{\partial V_{2}}\right) /\left|\frac{\partial Y_{T 2}}{\partial \omega_{2}}\right|^{2}
\end{aligned}
$$

The matrix in (23) contains two eigenvalues at zero, $\lambda_{3}=\lambda_{4}=0$, one associated with each of the two concurrent autonomous oscillations. The other two eigenvalues are:

$\lambda_{1,2}=\frac{a_{11}+a_{22}}{2} \pm \frac{\sqrt{\left(a_{11}+a_{22}\right)^{2}-4 D}}{2}$
$D=a_{11} a_{22}-a_{12} a_{21}$

For the quasi-periodic solution to be stable, the condition $\operatorname{Re}\left(\lambda_{1,2}\right)<0$ must be fulfilled, which implies $a_{11}+a_{22}<0$ and $D>0$. For $\left(a_{11}+a_{22}\right)^{2}-4 D<0$, the two poles will be complex-conjugate.

For illustration, the previous analysis will be applied to the case of a purely resistive nonlinearity $i(v)$. The stability conditions are:

$$
\begin{aligned}
& a_{T}=a_{11}+a_{22}=-\frac{\partial Y_{T 1}^{i}}{\partial \omega_{1}} \frac{\partial Y_{T 1}^{r}}{\partial V_{1}} /\left|\frac{\partial Y_{T 1}}{\partial \omega_{1}}\right|^{2}-\frac{\partial Y_{T 2}^{i}}{\partial \omega_{2}} \frac{\partial Y_{T 2}^{r}}{\partial V_{2}} /\left|\frac{\partial Y_{T 2}}{\partial \omega_{2}}\right|^{2}<0 \\
& D=K\left(\omega_{1}, \omega_{2}\right)\left(\frac{\partial Y_{T 1}^{r}}{\partial V_{1}} \frac{\partial Y_{T 2}{ }^{r}}{\partial V_{2}}-\frac{\partial Y_{T 1}^{r}}{\partial V_{2}} \frac{\partial Y_{T 2}^{r}}{\partial V_{1}}\right)>0
\end{aligned}
$$

where:

$K\left(\omega_{1}, \omega_{2}\right)=\frac{\partial Y_{T 1}^{i}}{\partial \omega_{1}} \frac{\partial Y_{T 2}^{i}}{\partial \omega_{2}} /\left(\left|\frac{\partial Y_{T 1}}{\partial \omega_{1}}\right|^{2}\left|\frac{\partial Y_{T 2}}{\partial \omega_{2}}\right|^{2}\right)$

Assuming positive values of $\partial Y_{T 1}^{i} / \partial \omega_{1}, \partial Y_{T 2}^{i} / \partial \omega_{2}$, for the quasi-periodic solution to be robust, the admittance function of each of the two concurrent oscillations should exhibit low sensitivity to the amplitude of the other one. In the particular case of $i(v)=a v+b v^{3}+d v^{5}$, the determinant $D$ in (25) is: 
$D=K\left(\omega_{1}, \omega_{2}\right)\left[\begin{array}{l}-27 b^{2}-150 d^{2}\left(V_{1}^{4}+V_{2}^{4}\right)-200 d^{2} V_{1}^{2} V_{2}^{2} \\ -120 b d\left(V_{1}^{2}+V_{2}^{2}\right)\end{array}\right] V_{1} V_{2}($

For $d=0$, the determinant in (27) particularizes to the one obtained in the case of a cubic nonlinearity. In that case, the stability condition $D>0$ cannot be fulfilled, in agreement with [13].

Fig. 3(b) presents the evolution of the real part of the poles of the quasi-periodic solutions in Fig. 2. The quasi-periodic solution generated from the mode at $f_{h}$ arises from an unstable periodic section and remains unstable for all the $d$ values. On the other hand, the quasi-periodic solution generated from the mode at $f_{d}$ is stable only in a small $d$ interval, between the two turning points of the quasi-periodic curve. In the transient simulations it has not been possible to obtain initial conditions (provided at a single circuit node) leading to this solution.

\section{Stability of the doubly-autonomous solution}

Assume a quasi-periodic regime arising from a periodic one at the frequency $\omega_{1}$. At the corresponding secondary Hopf bifurcation, one has $D=a_{11} a_{22}-a_{12} a_{21}=0$ in (25). This is because the circuit is in small-signal conditions with respect to the newly generated fundamental, and the derivatives $\partial Y_{T 1} / \partial V_{2}$ and $\partial Y_{T 2} / \partial V_{2}$ are equal to zero when calculated at $V_{2}=0$. Therefore, the two poles in (25) degenerate into $\lambda_{1}=0$ and $\lambda_{2}=a_{11}$. This is a general property, in the sense that all the doubly-autonomous quasi-periodic solutions will exhibit three poles at zero $\lambda_{1}=\lambda_{3}=\lambda_{4}=0$ at the secondary-Hopf bifurcation point where they are generated. At this point, the quasi-periodic solution is essentially the same as the periodic one, so this periodic solution should also exhibit the real pole $\lambda_{2}=a_{11}$. This real pole, associated with the resonator at $\omega_{1}$, is the one that would be obtained with a stability analysis of the periodic solution under the assumption of a small perturbation frequency $s$ [24],[33]. In addition to this pole, and for any parameter value, the periodic solution exhibits a pole at zero (due to its autonomy), and, in general, a pair of complexconjugate poles about $\omega_{2}$. These complex-conjugate poles are the ones that cross the imaginary axis at the secondary Hopf bifurcation, as shown in Fig. 2 and Fig. 3. This total number of poles is 4 , as in the quasi-periodic system (23).

When moving away from the secondary Hopf bifurcation (through the quasi-periodic solution path), the pole $\lambda_{1}$ will shift continuously from its original value $\lambda_{1}=0$, due to the continuity of the circuit equations. Therefore, our assumption of a small complex-frequency $s$ in the stability analysis of the quasi-periodic solution is well justified. From the bifurcation point, $\lambda_{1}$ may shift to the LHS or to the RHS. To predict this evolution of $\lambda_{1}$, one can calculate its derivatives with respect to the four state variables at the secondary Hopf bifurcation, where $V_{2}=0$, which depend implicitly on the analysis parameter. The increment undergone by $\lambda_{1}$ is given by:

$$
\Delta \lambda_{1}=\frac{\partial \lambda_{1}}{\partial V_{1}} \Delta V_{1}+\frac{\partial \lambda_{1}}{\partial V_{2}} \Delta V_{2}+\frac{\partial \lambda_{1}}{\partial \omega_{1}} \Delta \omega_{1}+\frac{\partial \lambda_{1}}{\partial \omega_{2}} \Delta \omega_{2}
$$

For convenience, we will use the dummy variable $X$ to obtain the derivatives in (28) at $V_{2}=0$, which are given by:

$$
\frac{\partial \lambda_{1}}{\partial X}=\frac{1}{a_{11}} \frac{\partial D}{\partial X}
$$

Because the system is in small signal conditions with respect to $\omega_{2}$, the only nonzero derivative is $\partial \lambda_{1} / \partial V_{2}$, as is easily derived from a simple inspection of (27). There are four cases:

i) $\lambda_{2}=a_{11}<0, \partial D / \partial V_{2}>0$, the newly-generated doubly autonomous solution is stable.

ii) $\lambda_{2}=a_{11}<0, \partial D / \partial V_{2}<0$, the new solution is unstable with one real pole on the RHS. This is the situation in the case of the analysis in Fig. 2 and Fig. 3(b).

iii) $\lambda_{2}=a_{11}>0, \partial D / \partial V_{2}>0$, the new solution is unstable, with two real poles on the RHS.

iv) $\lambda_{2}=a_{11}>0, \partial D / \partial V_{2}<0$, the new solution is unstable, with one real pole on the RHS.

Applying (29) in the particular case of $i(v)=a v+b v^{3}+d v^{5}$, one obtains:

$$
\frac{\partial \lambda_{1}}{\partial V_{2}}=\frac{K\left(\omega_{1}, \omega_{2}\right)}{a_{11}}\left(-\frac{75}{2} V_{1}^{5} d^{2}-30 V_{1}^{3} b d-\frac{27}{4} V_{1} b^{2}\right)
$$

Let $\lambda_{2}=a_{11}<0$ be considered. Assuming also positive values of $\partial Y_{1}^{i} / \partial \omega_{1}, \partial Y_{2}^{i} / \partial \omega_{2}$, for the pole $\lambda_{1}$, originally at zero, to shift to the LHS, the term in brackets, on the right side of (30) , should be positive. However, for $b<0, d>0$, this term is always negative. This is shown by solving the bi-quadratic equation:

$$
-\frac{75}{2} V_{1}^{4} d^{2}-30 V_{1}^{2} b d-\frac{27}{4} b^{2}=R
$$

where $R$ is a real number. The above equation only admits real solutions, $V_{1}^{2}$, for $R<0$, so the magnitude of the left-hand side expression must always be negative. Therefore, in the case of the polynomial model, the doubly autonomous quasi-periodic solutions will be unstable in the neighborhood of the secondaryHopf bifurcation.

When further modifying the parameter, and assuming that $\lambda_{2}$ remains in the LHS, the change in the sense of variation of $\lambda_{1}$, followed by its crossing to the LHS, would give rise to the stabilization of the doubly-autonomous quasi-periodic solution. The crossing of $\lambda_{1}$ through zero will be associated with a turning point in the quasi-periodic solution curve. This is the 
stabilization mechanism of the quasi-periodic solution arising from the periodic mode at $f_{d}$ in the diagram of Fig. 2 (see the small interval between the two turning points of the quasiperiodic solution). To increase the parameter interval of stable behavior, it will be helpful to obtain the turning-point locus in quasi-periodic regime, given by the following equation:

$$
T_{q p}\left(\eta_{1}, \eta_{2}\right)=a_{11} a_{22}-a_{12} a_{21}=D=0
$$

which must be resolved in combination with the steady-state system (15).

For illustration, the above turning-point locus has been superimposed in the plane defined by $R_{1}$ and $d$ (Fig. 4). The locus is closed, with a nearly triangular shape. To obtain a larger $d$ interval with stable quasi-periodic operation, the resistance $R_{1}$ has been reduced to $R_{1}=25 \Omega$. Fig. 5(a) presents the evolution of the steady-state solutions versus the parameter $d$. Fig. 5(b) presents the real part of the poles of the quasi-periodic solution traced versus $d$. There is a significant increase of the stable interval, though this stable quasi-periodic solution coexists with the stable oscillations at $f_{h}$ and $f_{d}$. When using time domain integration, the quasi-periodic solution is now easily reached, since a broad range of initial values lead to this solution, unlike the case of Fig. 2. The periodic and quasi-periodic spectra obtained with time-domain integration for the same value $d=8 \mathrm{~m} \Omega^{-1} / \mathrm{V}^{4}$ are shown in Fig. 5(c). For validation, solution points obtained with time-domain integration are superimposed with markers (crosses and circles - periodic solutions, diamonds and squares - quasi-periodic solutions) in Fig. 5(a).

In all our tests, the HB-based stability analysis [29-30] applied to doubly-autonomous quasi-periodic solutions has failed to provide any satisfactory results. This is attributed to the ill-conditioning of the conversion matrix in the presence of two Lyapunov exponents at zero [22]. This should lead to a large set of poles theoretically located on the imaginary axis, and corresponding to the harmonics of each of the two incommensurable frequencies and their intermodulation products $k \omega_{1}+m \omega_{2}$, where $k$ and $m$ are integer numbers. Some of these poles, of difficult distinction from the relevant poles (providing the actual stability information), may lie on the RHS, due to computational inaccuracies.

Use of (23)-(25) can alleviate this failure, since, as shown in Section IV, this analysis can be applied to practical oscillators using a semi-analytical technique, though some caution is needed.

In systems of dimension $N>4$, the analysis will be valid at least in the neighborhood of the secondary Hopf bifurcation, provided that the unstable subspace of the periodic solution has, at most, a dimension of one at this bifurcation point. Note that this subspace excludes the critical poles that cross the imaginary axis at the bifurcation point, which transform into two poles at zero of the quasi-periodic solution. Instead, the limitation is due to other unstable complex-conjugate poles of the periodic solution, which would be transferred to the quasi-periodic one. These poles cannot be detected with the two-dimension analysis in (25). They should be identified and stabilized through a circuit-level analysis of the periodic solution [30]-[31]. On the other hand, under the assumption of a load network such as the one considered in Fig. 1, with high quality factors $Q_{l}$ and $Q_{h}$, one can expect the two poles in (25) to be the dominant ones, due to the high values of the frequency derivatives in (24), which would place them near the imaginary axis

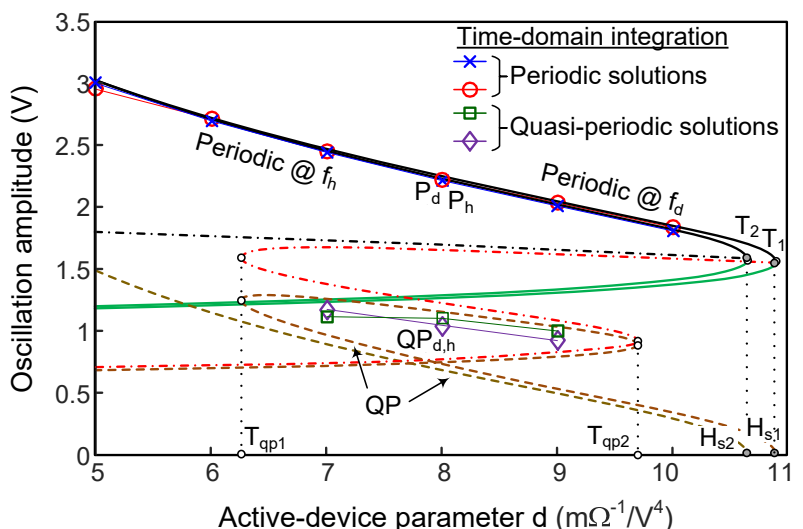

(a)

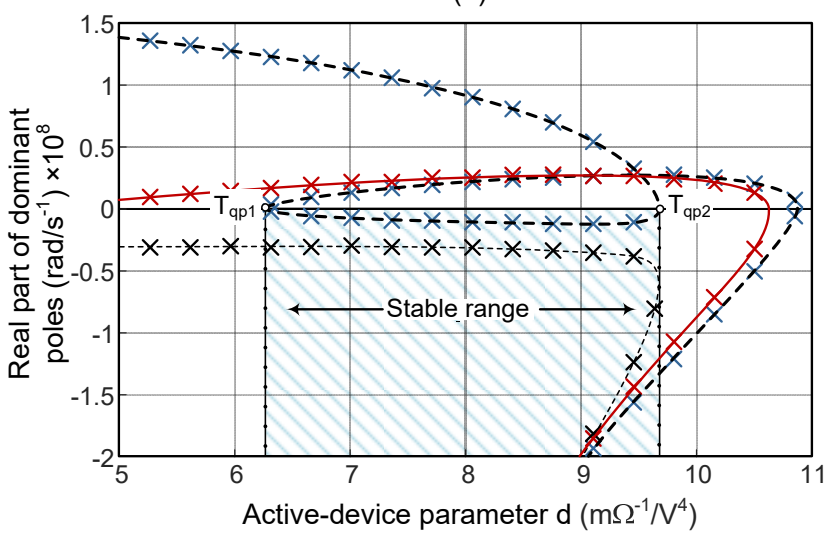

(b)
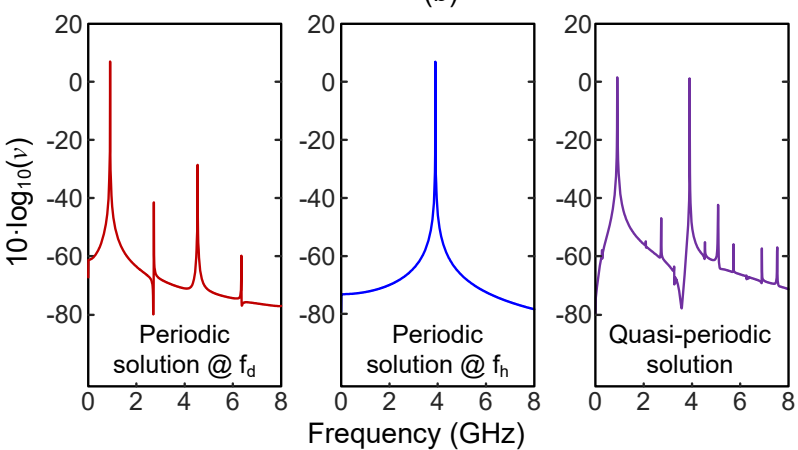

(c)

Fig. 5. Oscillator behavior for $R_{1}=25 \Omega$. (a) Bifurcation diagram versus the parameter $d$. The two components (at $f_{d}$ and $f_{h}$ ) of the quasi-periodic solution generated at $\mathrm{H}_{\mathrm{s} 1}$ and $\mathrm{H}_{\mathrm{s} 2}$ are traced in dash-dotted and dashed line. Solution points obtained with time-domain integration are superimposed with markers. (b) Stability analysis of the quasi-periodic solution. The real part of the poles is traced versus $d$. (c) Periodic and quasi-periodic spectra obtained with timedomain integration, using different initial conditions, for the same value $d=8 \mathrm{~m} \Omega^{-1} / \mathrm{V}^{4}$

\section{ANALYSIS OF A CROSS-COUPLED TRANSISTOR OSCILLATOR}

The investigation has been applied to the practical oscillator based on two cross-coupled transistors, shown in Fig. 1(b). The 
circuit has been designed for operation at the two frequencies $f_{d}=900 \mathrm{MHz}$ and $f_{h}=2.5 \mathrm{GHz}$. The analysis has been carried out with the aid of auxiliary generators (AGs) [24]-[25], connected in parallel, across the two transistors. Two values of the gate-bias voltage, leading to a qualitatively different behavior, are going to be considered: $V_{G S}=-0.7 \mathrm{~V}$ and $V_{G S}=-1.4 \mathrm{~V}$.

\section{A. Gate-bias voltage $V_{G S}=-0.7 \mathrm{~V}$}

Initially, the bias point $V_{G S}=-0.7 \mathrm{~V}$ has been considered, above the conduction threshold. In this situation, the circuit exhibits an excess of negative conductance in small signal (equivalent to $a<0$ ), so one can expect the existence of one or more primary Hopf loci. The analysis parameters will be $V_{D D}$ and the capacitor $C_{1}$, in the lower frequency resonator, which will be varied with varactor diodes.

\section{1) Hopf-bifurcation loci}

The primary Hopf bifurcation corresponding to the mode $\omega_{m}$ will be calculated using a single $\mathrm{AG}$, at the frequency $\omega_{A G}=\omega_{m}$, with a very small value $A_{A G}=\varepsilon$. The locus is obtained by solving:

$$
Y_{A G}\left(V_{D D}, C_{1}, \omega_{A G}\right)=0
$$

where $Y_{A G}$ is the ratio between the AG current and voltage. The above complex equation is solved in terms of $V_{D D}, C_{1}, \omega_{A G}$ through optimization in commercial software, with the pure HB system as an inner tier. Note that equation (33) is conceptually equivalent to (9). It provides a curve in the plane defined by $V_{D D}$ and $C_{1}$. The results are shown in Fig. 6. There are two different primary Hopf loci, associated with the onset of the periodic modes at $f_{d}=900 \mathrm{MHz}$ and $f_{h}=2.5 \mathrm{GHz}$, respectively. The Hopf loci intersect, which implies the existence of disjoint regions in which only one of the two periodic modes is stable.

Only oscillations generated from a stable $d c$ regime are originally stable or can become stable just after passing through a turning point. On the other hand, an oscillation generated from an unstable $d c$ regime will be originally unstable, but may become stable if it undergoes an inverse secondary Hopf bifurcation. Therefore, tracing the secondary-Hopf loci associated with each of the two oscillations will be essential to predict the parameter ranges in which each periodic mode exists as a single stable solution. This will be done by using two AGs, at the respective frequencies $\omega_{A G 1}, \omega_{A G 2}$. The AG at $\omega_{A G 1}$, with the amplitude $A_{A G 1}$, enables the calculation of the steadystate periodic oscillation at this frequency. The second AG at $\omega_{A G 2}$, with very small amplitude $A_{A G 2}=\varepsilon$ imposes a limit condition for the existence of the second fundamental frequency. The outer-tier system is:

$$
\begin{array}{r}
Y_{A G, 1}\left(A_{A G 1}, \omega_{A G 1}, V_{D D}, C_{1}\right)=0 \\
Y_{A G, 2}\left(A_{A G 1}, \omega_{A G 1}, \omega_{A G 2}, V_{D D}, C_{1}\right)=0
\end{array}
$$

which is resolved through optimization, with the pure HB system as an inner tier. System (34) is conceptually equivalent to the analytical system (10) and provides a curve in the plane $C_{1}, V_{D D}$. To avoid an excessive complexity, only the lower section of the loci (34), relevant to the first stabilization of the coexistent periodic solutions, has been represented in Fig. 6 . For the lower $C_{1}$ values, when increasing $V_{D D}$ from $2 \mathrm{~V}$, the oscillator initially undergoes a primary Hopf bifurcation, leading to the generation of a periodic solution at $f_{d}$. Then, it undergoes a second primary Hopf bifurcation leading to the generation of a periodic solution at $f_{h}$. The generation of this second periodic solution does not have any physical implications in the circuit behavior. This is because a solution generated from an unstable dc regime is initially unstable. Then, when crossing the secondary Hopf bifurcation locus (associated with $f_{h}$ ) a quasi-periodic regime at $f_{h}$ and $f_{d}$ is generated. As will be shown, this new regime is unstable, but the secondary Hopf bifurcation leads to the stabilization of the periodic oscillation at $f_{h}$, so it is relevant for the circuit physical behavior.

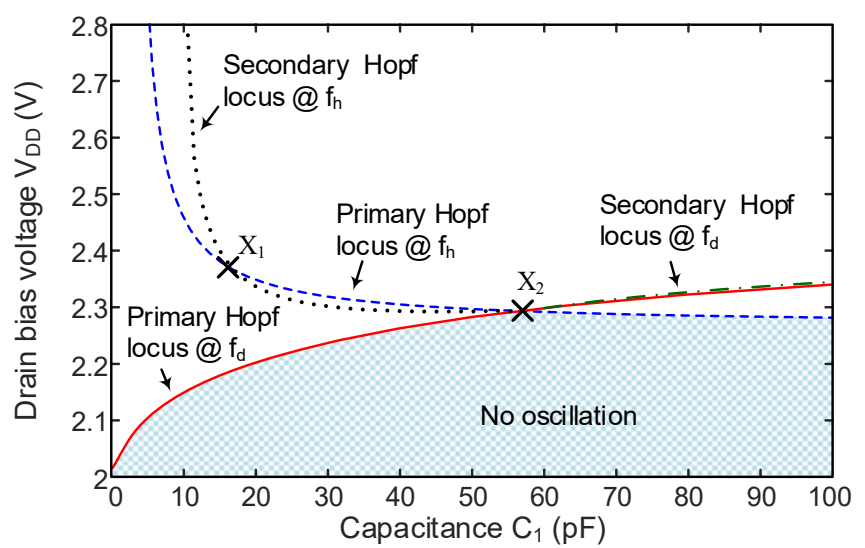

Fig. 6. Cross-coupled dual-frequency oscillator at the bias point $V_{G S}=-0.7 \mathrm{~V}$. Primary and secondary Hopf-bifurcation loci in the plane defined by $V_{D D}$ and $C_{1}$.

The primary Hopf locus and secondary Hopf locus associated with $f_{h}$ intersect at the point $X_{1}$. When increasing $V_{D D}$ for $C_{1}$ values above $X_{1}$, the secondary Hopf bifurcation occurs before the primary Hopf bifurcation. As seen in Fig. 7, which presents the solution curves obtained versus $V_{D D}$ for $C_{1}=20 \mathrm{pF}$, this is because the periodic solution curve at $f_{h}$ exhibits a turning point. Thus, it exists for $V_{D D}$ values lower than the one at which this periodic regime at $f_{h}$ is generated. At the point $X_{2}$, the secondary Hopf locus merges the two primary Hopf bifurcation loci. This corresponds to a degenerate solution of (34), with $A_{A G 1}=0$. In fact, the secondary Hopf locus vanishes at this intersection point. To the right of the point $X_{2}$, when increasing $V_{D D}$ from low value, the mode at $f_{h}$ starts-up from the stable $d c$ solution. It is the only stable one in the parameter region delimited by the primary Hopf locus associated to $f_{h}$ and the secondary Hopf locus associated to $f_{d}$. In this particular case the primary and secondary Hopf loci of the mode at $f_{d}$ are nearly overlapped. To summarize, below (above) the intersection point $X_{2}$, the periodic solution at $f_{d}\left(f_{h}\right)$ starts from the $d c$ regime and is the only stable one in a certain parameter interval. This behavior has been validated through experimental measurements. The solution spectrum has been measured extracting the signal from one of the drain nodes with a lossy coupled-line coupler. Fig. 8 shows the spectrum before the onset of each of the two periodic 
oscillations, the spectrum just after the bifurcation and the evolution of each of these two periodic oscillations versus $V_{D D}$. In agreement with Fig. 6 , for different values of the $C_{1}$, there are disjoint parameter intervals in which only one of the two periodic modes has been physically observed in the measurements.

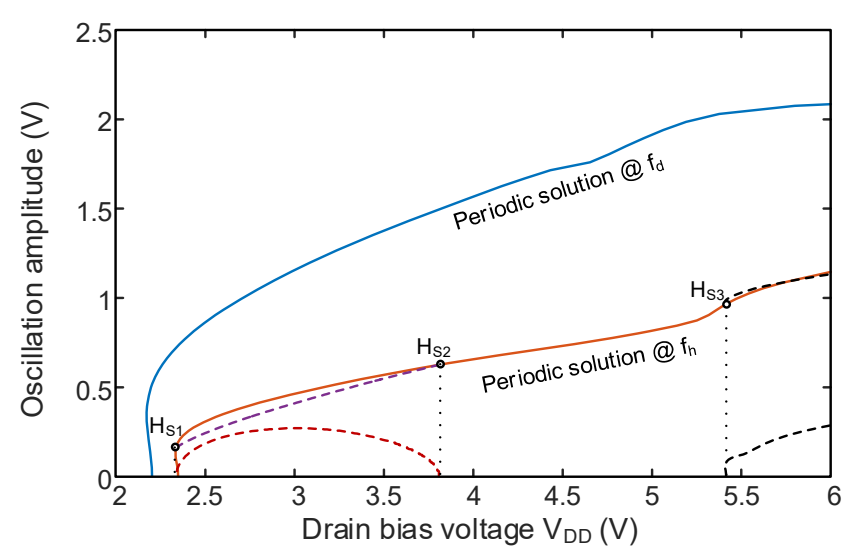

Fig. 7. Cross-coupled dual-frequency oscillator at the bias point $V_{G S}=-0.7 \mathrm{~V}$. Bifurcation diagram versus the parameter $V_{D D}$ for fixed capacitor values $C_{1}=20$ $\mathrm{pF}$ and $C_{2}=0.676 \mathrm{pF}$.
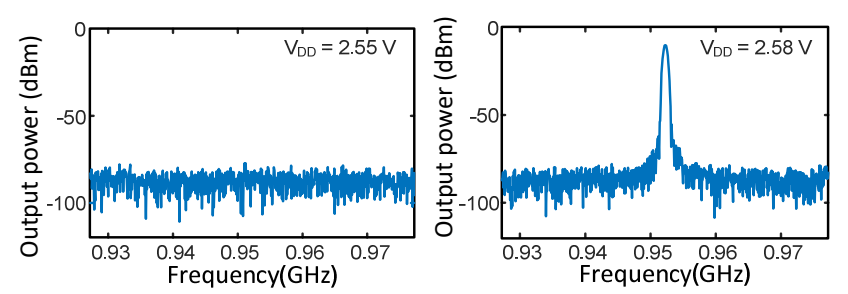

(a)

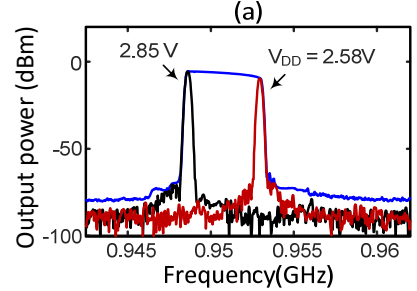

(c)

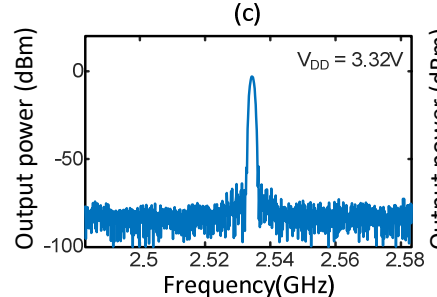

(e)

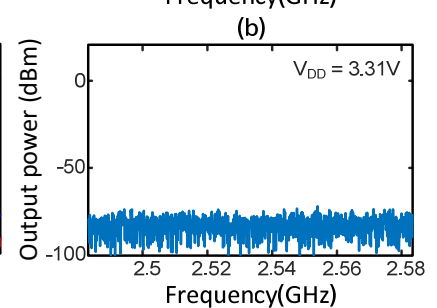

(d)

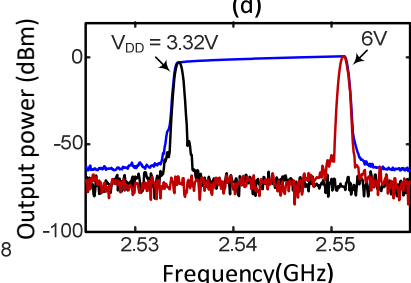

(f)

Fig. 8. Experimental measurements of the two periodic modes for two values of $\mathrm{C}_{1}$. For each value of $\mathrm{C}_{1}$, only one of the modes is observed. (a) Spectrum before the onset of the mode at $f_{d}$. (b) Spectrum just after the generation of the mode at $f_{d}$. (c) Evolution of this mode under variations in $V_{D D}$. Measured spectra in the interval $2.58 \mathrm{~V} \leq V_{D D} \leq 2.85 \mathrm{~V}$ are shown. (d) Spectrum before the onset of the mode at $f_{h}$. (e) Spectrum just after the generation of the mode at $f_{h}$. (f) Evolution of this mode under variations in $V_{D D}$. Measured spectra in the interval $3.32 \mathrm{~V} \leq V_{D D} \leq 6 \mathrm{~V}$ are shown.

\section{2) Bifurcation diagram versus $V_{D D}$}

Next, the whole bifurcation diagram is traced versus $V_{D D}$ for fixed capacitor values $C_{1}=20 \mathrm{pF}$ and $C_{2}=0.676 \mathrm{pF}$ (Fig. 7). The periodic solutions detected with (33) have been calculated with a single AG [24]-[25]. The periodic solution at $f_{d}$ arises from a stable dc regime, whereas the periodic solution at $f_{h}$ arises from an unstable $d c$ regime, with a pair of complex-conjugate poles on the RHS. In agreement with the predictions of Fig. 6, the solution at $f_{h}$ undergoes a secondary Hopf bifurcation $\left(\mathrm{H}_{\mathrm{s} 1}\right)$ for a $V_{D D}$ value lower than the one at which it is generated. A quasiperiodic solution is generated at $\mathrm{H}_{\mathrm{s} 1}$. Then, the periodic solution at $f_{h}$ undergoes two additional secondary Hopf bifurcations: $\mathrm{H}_{\mathrm{s} 2}$, at which the quasi-periodic solution is extinguished, and $\mathrm{H}_{\mathrm{s} 3}$, at which a quasi-periodic solution arises again. These steady-state quasi-periodic solutions (also shown in Fig. 7) are calculated by solving the following outer-tier system:

$$
\begin{gathered}
Y_{A G, 1}\left(A_{A G 1}, A_{A G 2}, \omega_{A G 1}, \omega_{A G 2}, V_{D D}\right)=0 \\
Y_{A G, 2}\left(A_{A G 1}, A_{A G 2}, \omega_{A G 1}, \omega_{A G 2}, V_{D D}\right)=0
\end{gathered}
$$

where the pure HB system acts as an inner tier.

\section{3) Stability analysis of the periodic solutions}

The stability of the periodic curves in Fig. 7 has been analyzed with pole-zero identification [30]-[31]. The periodic solution at $f_{d}$ is always stable. Regarding the solution at $f_{h}$, Fig. 9(a) presents the variation of the real part of the complexconjugate poles at $f_{d}$, represented versus $V_{D D}$. In view of this plot, the periodic solution at $f_{h}$ is initially unstable, with a par of complex-conjugate poles at $f_{d}$ on the RHS. These poles cross to the LHS at $\mathrm{H}_{\mathrm{S} 1}$, which gives rise to the stabilization of the periodic solution at $f_{h}$ and to the generation of a quasi-periodic solution at $f_{d}$ and $f_{h}$. This quasi-periodic solution is extinguished at the Hopf bifurcation $\mathrm{H}_{\mathrm{s} 2}$, from which the periodic solution at $f_{h}$ becomes unstable. The pair of poles at $f_{d}$ crosses again to the RHS at $\mathrm{H}_{\mathrm{s} 3}$, which leads to a new stabilization of the solution at $f_{h}$, plus the onset of a new quasi-periodic regime at $f_{h}$ and $f_{d}$.

\section{4) Stability analysis of the quasi-periodic solution}

The method based on pole-zero identification has failed to predict the stability properties of the doubly-autonomous quasiperiodic solution. It provided poles on the RHS for solution points in both the upper and lower sections of the quasi-periodic solution curve in Fig. 10(a). However, for $4.3 \mathrm{~V}<V_{D D}<5.1 \mathrm{~V}$, it was possible to obtain quasi-periodic solutions with timedomain integration and in measurements. As already stated, the failure is not attributed to the pole-zero identification method, but to the ill-conditioning of the conversion matrix in the presence of two Lyapunov exponents at zero [22]. Instead, the stability of these quasi-periodic solutions has been studied in a semi-analytical manner, calculating the derivatives in (24) with the aid of the same two AGs used for the steady-state calculation. This is done numerically, by applying finite differences to these two AGs, in a sequential manner [34]. The analysis has been applied to the two quasi-periodic solutions presented in Fig. 7. Fig. 9(b) shows the evolution of the poles 
$\lambda_{1}$ and $\lambda_{2}$ through the first quasi-periodic solution, comprised between $\mathrm{H}_{\mathrm{s} 1}$ and $\mathrm{H}_{\mathrm{s} 2}$. At these two bifurcations the pole $\lambda_{1}$ takes a zero value, in agreement with the discussions in Section III. Between $\mathrm{H}_{\mathrm{s} 1}$ and $\mathrm{H}_{\mathrm{s} 2}$, it takes a positive value, which indicates that the whole quasi-periodic solution curve is unstable. On the other hand, the pole $\lambda_{2}$ is negative, with small magnitude at $\mathrm{H}_{\mathrm{s} 1}$, in consistency with the proximity of this bifurcation to the turning point of the periodic curve at $f_{d}$. It keeps negative through the whole quasi-periodic solution curve. With the same kind of analysis, it has been verified that the quasi-periodic solution generated at $\mathrm{H}_{\mathrm{s} 3}$ is also unstable. The two cases correspond to case ii) of the classification given in Section III.C.

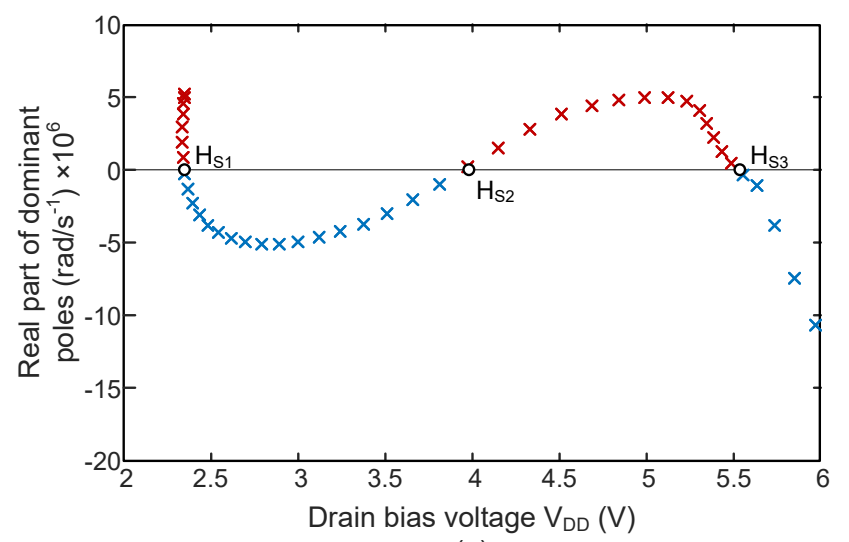

(a)

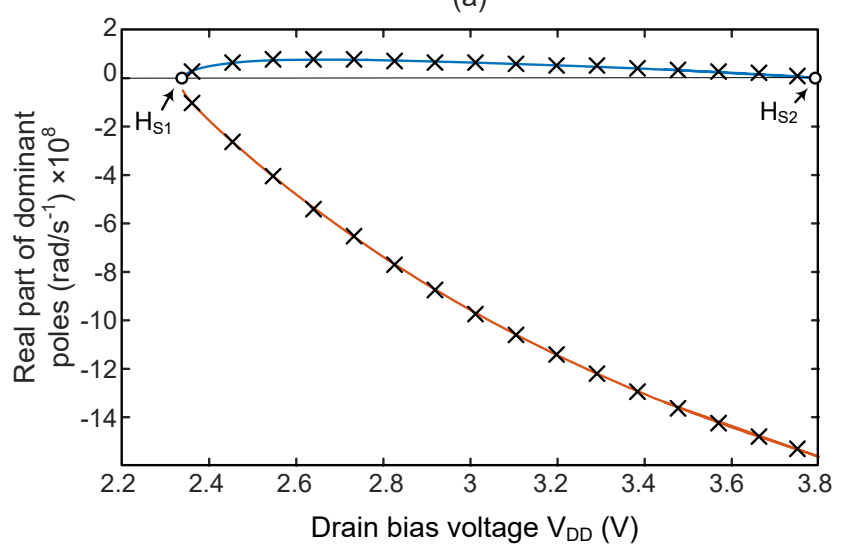

(b)

Fig. 9. Cross-coupled dual-frequency oscillator at the bias point $V_{G S}=-0.7 \mathrm{~V}$. (a) Stability analysis of the periodic curve at $f_{h}$ versus $V_{D D}$. (b) Stability analysis of the quasi-periodic solution using (23) to (25).

\section{B. Gate-bias voltage $V_{G S}=-1.4 \mathrm{~V}$}

Next, the bias point $V_{G S}=-1.4 \mathrm{~V}$, below the conduction threshold, will be considered. In this case, the circuit does not exhibit negative resistance in small signal for low and moderate $V_{D D}$ values. However, when increasing the oscillation amplitude, there is an increase in negative conductance, followed by a reduction, in a manner similar to what happens with the polynomial model for $a>0, b<0, d>0$. Fig. 10(a) shows the bifurcation diagram versus $V_{D D}$. The two periodic solution curves are only generated from $d c$ regime for sufficiently high $V_{D D}$, and exhibit turning points $\left(\mathrm{T}_{1}\right.$ and $\left.\mathrm{T}_{2}\right)$ for similar oscillation amplitudes. The oscillation at $f_{d}$ arises before the one at $f_{h}$ and is the only one generated from a stable $d c$ regime.

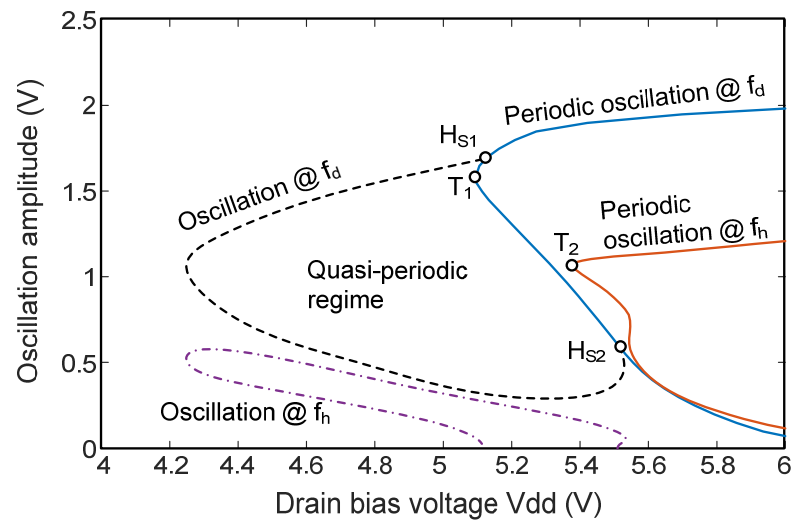

(a)

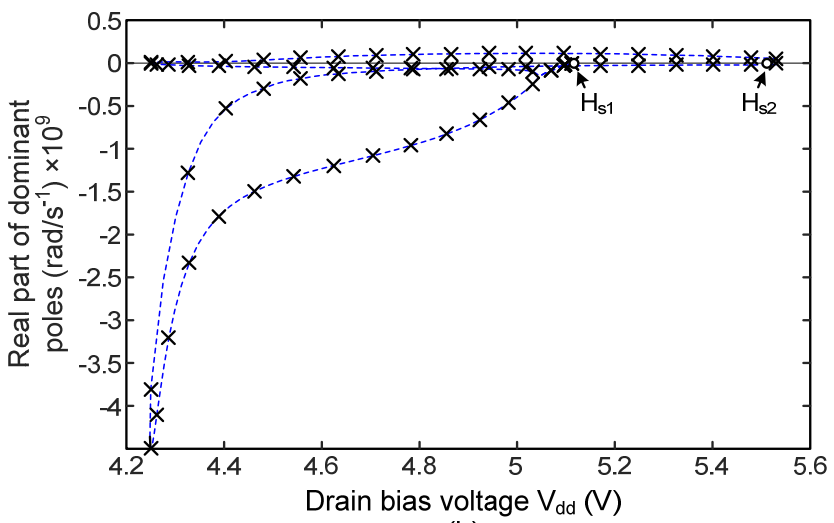

(b)

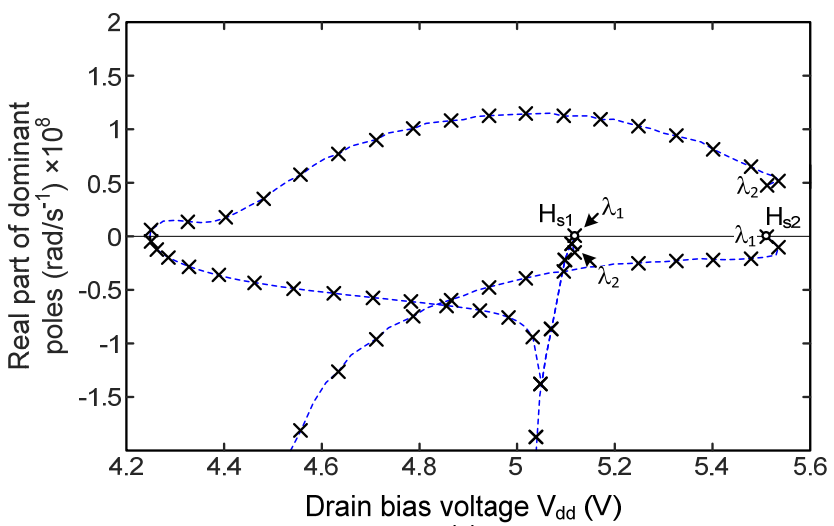

(c)

Fig. 10. Cross-coupled dual-frequency oscillator at the bias point $V_{G S}=-1.4 \mathrm{~V}$ and capacitor value $C_{1}=55 \mathrm{pF}$. (a) Bifurcation diagram versus $V_{D D}$. (b) Stability analysis of the quasi-periodic solution generated from the periodic solution curve at $f_{d}$, and comprised between $\mathrm{H}_{\mathrm{s} 1}$ and $\mathrm{H}_{\mathrm{s} 2}$, using (23) to (25). (c) Expanded view of (b), showing the evolution of the poles in a greater detail. 
At the point $\mathrm{H}_{\mathrm{s}}$, very near the turning point $\mathrm{T}_{1}$ of the periodic curve at $f_{d}$, a secondary Hopf bifurcation takes place and gives rise to a doubly autonomous quasi-periodic regime at $f_{d}$ and $f_{h}$. The Hopf bifurcation $\mathrm{H}_{\mathrm{s} 1}$ arises from a stable periodic solution when reducing $V_{D D}$. The poles associated with the generated quasi-periodic solution are shown in Fig. 10(b) and in the expanded view of Fig. 10(c). The real part of the poles $\lambda_{1}$ and $\lambda_{2}$ has been represented versus $V_{D D}$. At the secondary Hopf bifurcation $\mathrm{H}_{\mathrm{s} 1}$, occurring from a stable periodic solution, the generated quasi-periodic regime (with amplitude at $f_{h}$ tending to zero at $\mathrm{H}_{\mathrm{s} 1}$ ), exhibits two real poles, one is $\lambda_{1}=0$ and the other fulfils $\lambda_{2}<0$ [Fig. 10(c)]. This Hopf bifurcation corresponds to case i) of the classification derived in Section III.C. When decreasing $V_{D D}$, the two real poles $\lambda_{1}$ and $\lambda_{2}$ immediately merge into a pair of complex-conjugate poles, at $V_{D D}=5.113 \mathrm{~V}$ [Fig. 10(c)]. Through this upper section of the quasi-periodic solution curve [Fig. 10(a)], the complexconjugate poles keep on RHS and split again into two real poles at $V_{D D}=5.051 \mathrm{~V}$ [Fig. $\left.10(\mathrm{c})\right]$. At $V_{D D}=4.245 \mathrm{~V}$, the real pole $\lambda_{2}$ crosses the imaginary axis to the RHS. When this crossing takes place, the quasi-periodic solution curve exhibits an infinite slope [Fig. 10(a)]. Through the lower section of the quasi-periodic curve, the pole $\lambda_{2}$ continues to shift rightwards on the RHS, then turns and is still positive when the quasiperiodic curve reaches the secondary Hopf bifurcation $\mathrm{H}_{\mathrm{s} 2}$ [Fig. 10(c)]. The pole $\lambda_{1}$ is negative through the entire lower section of the curve but tends to zero at $\mathrm{H}_{\mathrm{s} 2}$. At this bifurcation point, the component at $f_{d}$ of the quasi-periodic solution merges the unstable section of the periodic solution curve at $f_{d}$ (below the turning point $\mathrm{T}_{1}$ ). In fact, the pole $\lambda_{2}$ of the quasi-periodic solution comes from the (unstable) dominant real pole of the periodic one, in agreement with the derivations of Section III.C. The transformation in $\mathrm{H}_{\mathrm{s} 2}$ corresponds to case iii).

Unlike the situation obtained with the polynomial model in Section III.C and Fig. 5, the quasi-periodic solution arising at $\mathrm{H}_{\mathrm{s} 1}$ does not coexist with any of the two periodic solutions. Note that when the transistor is biased below threshold, oscillations cannot start from the $d c$ regime for most $V_{D D}$ values. However, once the oscillation has started, one can reduce the bias voltage while still maintaining the oscillation. In the case of periodic oscillations this is because the transistor conducts for a certain fraction of the oscillation period. In comparison, the "off" time intervals will be shorter in the case of the doubly-autonomous solutions, due to the irregularity of the quasi-periodic waveform. The behavior predicted by the analysis in Fig. 10 has been verified experimentally. Fig. 11 shows the spectra measured when reducing $V_{D D}$. For sufficiently high $V_{D D}$, a periodic spectrum is observed. When reducing this parameter, the quasi-periodic solution is generated at $V_{D D}=5.11 \mathrm{~V}$. This quasi-periodic solution does not coexist with any of the stable periodic modes. It is extinguished when further reducing $V_{D D}$.

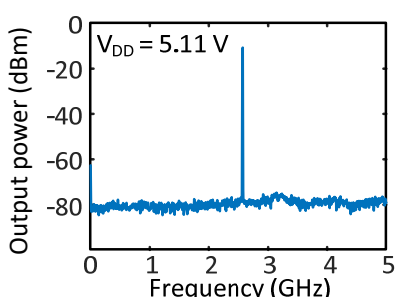

(a)

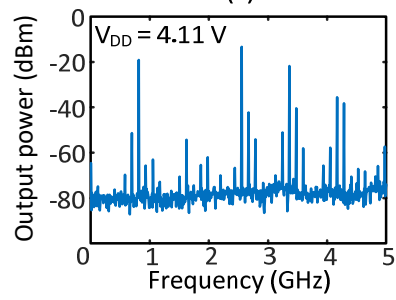

(c)

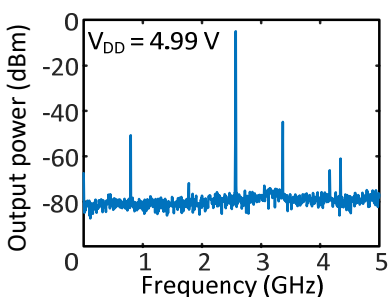

(b)

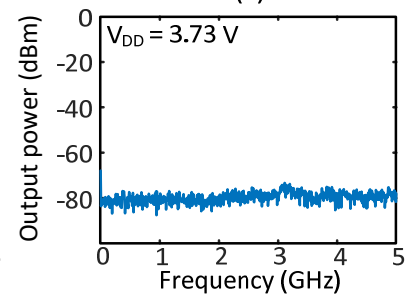

(d)
Fig. 11. Spectra measured when reducing $V_{D D}$. (a) For $V_{D D} \geq 5.11 \mathrm{~V}$ a periodic spectrum is observed. (b),(c) when further reducing this parameter, the quasiperiodic solution is generated. (d) In agreement with the results of Fig. 10(a), no oscillation exists for lower $V_{D D}$ values.

\section{SYNCHRONIZED OSCILLATIONS}

If the two coexistent oscillation frequencies synchronize [1112], they will exhibit a constant rational ratio, of the form: $\omega_{1} / \omega_{2}=M / N$, where $M$ and $N$ are positive integers. As a result of this rational ratio, there will be a constant phase relationship between the two oscillations.

\section{A. Analytical investigation}

For illustration, the case $\omega_{1} / \omega_{2}=1 / 3$ will be studied, of particular interest in practical oscillators [10]. For the analytical investigation, the polynomial model $i(v)=a v+b v^{3}+d v^{5}$ will be considered. Assuming a synchronized behavior of the two independent oscillations, the steady-state voltage will be represented as:

$$
v(t)=V_{1} \cos (\omega t)+V_{2} \cos (3 \omega t+\phi)
$$

where the phase origin is arbitrarily fixed at $\omega$. The describing function at $\omega$ is given by:

$$
\begin{aligned}
& Y_{1}\left(V_{1}, V_{2}, \phi\right)=a+\frac{3}{4} b V_{1}^{2}+\frac{5}{8} d V_{1}^{4}+\frac{3}{2} b V_{2}^{2}+\frac{15}{4} d V_{1}^{2} V_{2}^{2}+\frac{15}{8} d V_{2}^{4}+ \\
& \left(\frac{3}{4} b V_{2} V_{1}+\frac{5}{4} d V_{2} V_{1}^{3}+\frac{15}{8} d V_{1} V_{2}^{3}\right) e^{j \phi}+\frac{5}{16} d V_{2} V_{1}^{3} e^{-j \phi}
\end{aligned}
$$


In turn, the describing function at $3 \omega$ is given by:

$$
\begin{aligned}
& Y_{2}\left(V_{1}, V_{2}, \phi\right)=\left(\frac{5}{4} d V_{1}^{3} V_{2}+\frac{1}{4} b \frac{V_{1}^{3}}{V_{2}}+\frac{5}{16} d \frac{V_{1}^{5}}{V_{2}}\right) e^{-j \phi}+ \\
& \left(a+\frac{3}{4} b V_{2}^{2}+\frac{5}{8} d V_{2}^{4}+\frac{15}{4} d V_{1}^{2} V_{2}^{2}+\frac{3}{2} b V_{1}^{2}+\frac{15}{8} d V_{1}^{4}\right)+ \\
& \frac{5}{8} d V_{1}^{3} V_{2} e^{j \phi}
\end{aligned}
$$

The describing functions in (37) and (38), are no longer real, as a result of the phase dependence. The steady-state equations are given by:

$$
\begin{aligned}
& Y_{T 1}=Y_{1}\left(V_{1}, V_{2}, \phi\right)+Y_{L}(j \omega)=0 \\
& Y_{T 2}=Y_{2}\left(V_{1}, V_{2}, \phi\right)+Y_{L}(j 3 \omega)=0
\end{aligned}
$$

which, after splitting into real and imaginary parts, provides a system of four real equations in the four unknowns: $\left(V_{1}, V_{2}, \phi, \omega\right)$. Fig. 12 presents the synchronized solution of the circuit in Fig. 1(a) (with $C_{1}=44.2 \mathrm{pF}$, to facilitate this synchronization). Due to the intricate shape of the solution curve, it has been necessary to apply the arc-length continuation method [35] to obtain the whole solution curve.

The stability of the solutions of system (39) is analyzed considering a small-amplitude perturbation of complex frequency, which will involve the generalization of the characteristic system (19) to the $3^{\text {rd }}$ order. Under the more restrictive assumption of a small perturbation frequency s, one may take into account that this frequency increment acts like a time differentiator, which provides the perturbed system:

$$
\begin{gathered}
\frac{\partial Y_{T 1}^{i}}{\partial \omega_{1}} \frac{\Delta \dot{V}_{1}}{V_{1 o}}+\frac{\partial Y_{T 1}^{r}}{\partial \omega_{1}} \Delta \dot{\phi}_{1}=-\frac{\partial Y_{T 1}^{r}}{\partial V_{1}} \Delta V_{1}-\frac{\partial Y_{T 1}^{r}}{\partial V_{2}} \Delta V_{2}+\frac{\partial Y_{T 1}^{r}}{\partial \phi_{1}} \Delta \phi_{1}+\frac{\partial Y_{T 1}^{r}}{\partial \phi_{2}} \Delta \phi_{2} \\
-\frac{\partial Y_{T 1}^{r}}{\partial \omega_{1}} \frac{\Delta \dot{V}_{1}}{V_{1 o}}+\frac{\partial Y_{T 1}^{i}}{\partial \omega_{1}} \Delta \dot{\phi}_{1}=-\frac{\partial Y_{T 1}^{i}}{\partial V_{1}} \Delta V_{1}-\frac{\partial Y_{T 1}^{i}}{\partial V_{2}} \Delta V_{2}+\frac{\partial Y_{T 1}^{i}}{\partial \phi_{1}} \Delta \phi_{1}+\frac{\partial Y_{T 1}^{i}}{\partial \phi_{2}} \Delta \phi_{2} \\
\frac{\partial Y_{T 2}^{i}}{\partial \omega_{2}} \frac{\Delta \dot{V}_{2}}{V_{2 o}}+\frac{\partial Y_{T 2}^{r}}{\partial \omega_{2}} \Delta \dot{\phi}_{2}=-\frac{\partial Y_{T 2}^{r}}{\partial V_{1}} \Delta V_{1}-\frac{\partial Y_{T 2}^{r}}{\partial V_{2}} \Delta V_{2}+\frac{\partial Y_{T 2}^{r}}{\partial \phi_{1}} \Delta \phi_{1}+\frac{\partial Y_{T 2}^{r}}{\partial \phi_{2}} \Delta \phi_{2} \\
-\frac{\partial Y_{T 2}^{r}}{\partial \omega_{2}} \frac{\Delta \dot{V}_{2}}{V_{2 o}}+\frac{\partial Y_{T 2}^{i}}{\partial \omega_{2}} \Delta \dot{\phi}_{2}=-\frac{\partial Y_{T 2}^{i}}{\partial V_{1}} \Delta V_{1}-\frac{\partial Y_{T 2}^{i}}{\partial V_{2}} \Delta V_{2}+\frac{\partial Y_{T 2}^{i}}{\partial \phi_{1}} \Delta \phi_{1}+\frac{\partial Y_{T 2}^{i}}{\partial \phi_{2}} \Delta \phi_{2}
\end{gathered}
$$

The above Linear-Time-Invariant system can be rewritten in the following form:

$$
\left[\begin{array}{c}
\Delta \dot{V}_{1} \\
\Delta \dot{V}_{2} \\
\Delta \dot{\phi}_{1} \\
\Delta \dot{\phi}_{2}
\end{array}\right]=[M]\left[\begin{array}{l}
\Delta V_{1} \\
\Delta V_{2} \\
\Delta \phi_{1} \\
\Delta \phi_{2}
\end{array}\right]
$$

The poles of the synchronized solution will agree with the four eigenvalues of the matrix $[M]$. Because of the synchronized behavior of the two oscillations, this matrix will only have one eigenvalue at zero $\left(\lambda_{1}=0\right)$. The above eigenvalue analysis has been applied to the synchronized solution curve in Fig. 12. Only the sections in solid line correspond to stable behaviour and are delimited by turning points. In fact, turning points of ordinary injection-locked oscillators may correspond [36] either to jumps or desynchronization. The same behaviour is obtained in the case of two synchronized self-generated oscillations. The turning points $T_{1}$ and $T_{2}$ correspond to jumps from which the circuit evolves to the solution in the upper stable section. The turning points $T_{3}$ and $T_{4}$ are desynchronization points from which the circuit evolves to a quasi-periodic regime. Results from transient simulations are superimposed with asterisks.

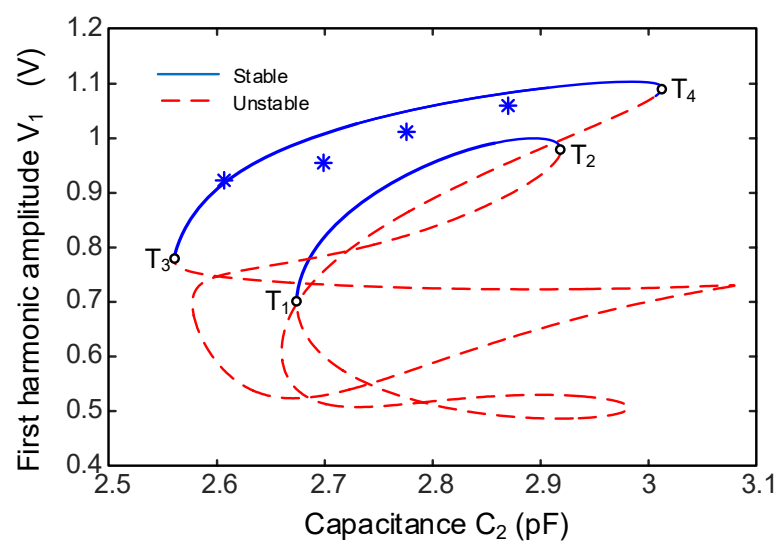

Fig. 12. Synchronized oscillations in the circuit of Fig. 1(a). The amplitudes $V_{1}$ and $V_{2}$ at the two oscillation frequencies have been represented versus the capacitor $C_{2}$. Only the curve sections in solid line are stable.

\section{B. Cross-coupled oscillator}

The analysis of the synchronized oscillation in HB will be carried out using two AGs, one at each oscillation frequency, fulfilling $\omega_{A G 2}=3 \omega_{A G 1}$. Because of the phase relationship between the two oscillations, the phase of one of the AGs, $\phi_{A G 2}$ , will be an unknown of the problem. The other phase variable may be arbitrarily set to zero, $\phi_{A G 1}=0$. The steady-state solution will be calculated by solving the system:

$$
\begin{aligned}
& Y_{A G, 1}\left(A_{A G 1}, A_{A G 2}, \omega_{A G 1}, \phi_{A G 2}, V_{D D}\right)=0 \\
& Y_{A G, 2}\left(A_{A G 1}, A_{A G 2}, \omega_{A G 1}, \phi_{A G 2}, V_{D D}\right)=0
\end{aligned}
$$

which is done through optimization with the pure HB system as an inner tier. Application to the oscillator in Fig. 1(b) provides the synchronized solution curve in Fig. 13(a), with a shape similar to the one in Fig. 12. The stability analysis has been carried out with standard pole-zero identification [30]-[31], which is applicable in this case due to the periodic nature of the solution. The upper section of the curve is stable, whereas the lower section is unstable. Fig. 13 includes the measurement results for similar conditions. Fig. 13(b) presents the spectrum just before the synchronization takes place. Fig. 13(c) presents the spectrum after this synchronization. 


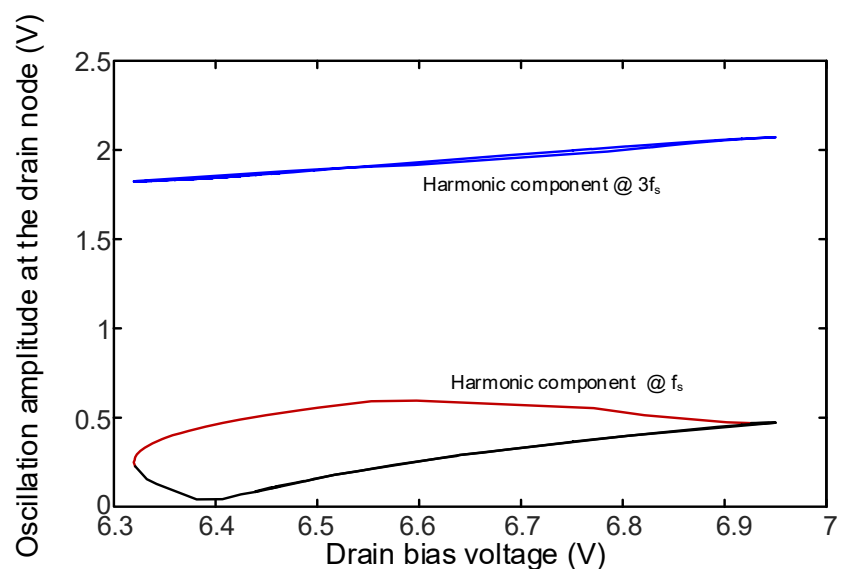

(a)

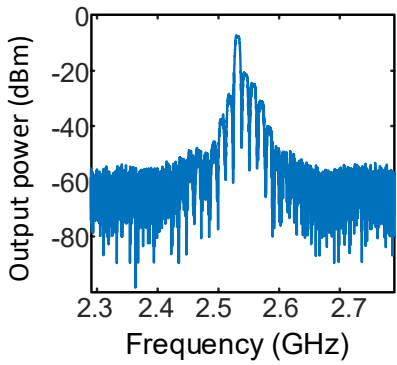

(b)

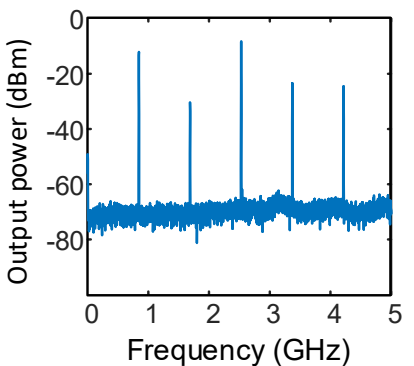

(c)
Fig. 13. Synchronization of the two individual oscillation signals. (a) Solution curve. (b) Experimental spectrum just before the synchronization takes place. (c) Experimental spectrum after synchronization.

\section{Phase noise}

One advantage of the synchronized operation of the two oscillations is the potential reduction of the phase-noise spectral density. To illustrate this, a white-noise current noise $I_{N}(t)$ of spectral density $N$, connected in parallel with the active device, will be considered. The effect of this noise source will be analysed including the real and imaginary parts of the instantaneous noise admittances (one at each of the two oscillation frequencies) on the right side of (41). However, the derivation of the phase-noise spectral density leads to cumbersome expressions. To get insight into the phase-noise behavior, the frequency variation of the load conductance will be neglected, as well as the system dependence on the amplitude perturbations. This leads to the following simplified system:

$$
\begin{aligned}
& \frac{\partial Y_{T 1}^{i}}{\partial \omega} \Delta \dot{\phi}_{1}=\frac{\partial Y_{T 1}^{i}}{\partial \phi_{1}} \Delta \phi_{1}+\frac{\partial Y_{T 1}^{i}}{\partial \phi_{2}} \Delta \phi_{2}+\frac{I_{N 1}^{i}}{V_{o 1}} \\
& \frac{\partial Y_{T 2}^{i}}{\partial \omega} \Delta \dot{\phi}_{2}=\frac{\partial Y_{T 2}^{i}}{\partial \phi_{1}} \Delta \phi_{1}+\frac{\partial Y_{T 2}^{i}}{\partial \phi_{2}} \Delta \phi_{2}+\frac{I_{N 2}^{i}}{V_{o 2}}
\end{aligned}
$$

where $I_{N 1}^{i}, I_{N 2}^{i}$ refer to the imaginary part of the instantaneous noise current. The system autonomy provides the following condition:
$Y_{T k}^{i}\left(\phi_{1}+\alpha, \phi_{2}+\alpha\right)=0, \quad \forall \alpha, \quad k=1,2 \rightarrow\left|\begin{array}{ll}\frac{\partial Y_{T 1}^{i}}{\partial \phi_{1}} & \frac{\partial Y_{T 1}^{i}}{\partial \phi_{2}} \\ \frac{\partial Y_{T 2}^{i}}{\partial \phi_{1}} & \frac{\partial Y_{T 2}^{i}}{\partial \phi_{2}}\end{array}\right|=0$

Now, applying (44), calculating the Fourier transform and taking into account that $I_{N 1}^{i}$ and $I_{N 1}^{i}$ are uncorrelated, one obtains the following expressions for the phase-noise spectral densities of the two oscillations:

$$
\begin{aligned}
&\left|\phi_{1}(\Omega)\right|^{2}= \frac{\Omega^{2}\left(\frac{\partial Y_{T 2}^{i}}{\partial \omega}\right)^{2}+\left(\frac{\partial Y_{T 2}^{i}}{\partial \phi_{2}}\right)^{2}+\left(\frac{\partial Y_{T 1}^{i}}{\partial \phi_{2}}\right)^{2}}{\Omega^{4}\left(\frac{\partial Y_{T 2}^{i}}{\partial \omega}\right)^{2}\left(\frac{\partial Y_{T 2}^{i}}{\partial \omega}\right)^{2}+\Omega^{2}\left(\frac{\partial Y_{2}^{i}}{\partial \phi_{2}} Y_{1 \omega}^{i}+\frac{\partial Y_{1}^{i}}{\partial \phi_{1}} Y_{2 \omega}^{i}\right)^{2}} \frac{2 N}{V_{o 1}^{2}} \\
&\left|\phi_{2}(\Omega)\right|^{2}=\frac{\Omega^{2}\left(\frac{\partial Y_{T 2}^{i}}{\partial \omega}\right)^{2}+\left(\frac{\partial Y_{T 1}^{i}}{\partial \phi_{1}}\right)^{2}+\left(\frac{\partial Y_{T 2}^{i}}{\partial \phi_{1}}\right)^{2}}{\Omega^{4}\left(\frac{\partial Y_{T 2}^{i}}{\partial \omega}\right)^{2}\left(\frac{\partial Y_{T 2}^{i}}{\partial \omega}\right)^{2}+\Omega^{2}\left(\frac{\partial Y_{2}^{i}}{\partial \phi_{2}} Y_{1 \omega}^{i}+\frac{\partial Y_{1}^{i}}{\partial \phi_{1}} Y_{2 \omega}^{i}\right)^{2}} \frac{2 N}{V_{o 1}^{2}}
\end{aligned}
$$

Obviously, the positive terms involving the phase derivatives will have a relevant effect on the phase-noise spectrum and may give rise to a reduction of the spectral density, in comparison with the one obtained in a free-running regime. The phase-noise in synchronized-concurrent periodic mode has been simulated with a semi-analytical method, based on the introduction of noise sources in (40), which extends [37] to this kind of autonomous regime. The various derivatives in (40) have been calculated applying finite differences to the two AGs that are used to obtain the synchronized-concurrent steady state [34], [37]. Fig. 14 shows the measured and simulated spectra corresponding to the two individual periodic oscillations at $f_{d}$ and $f_{h}$ and the synchronized-concurrent periodic solution, which exhibits a lower phase noise spectral density in a relatively large interval of offset frequencies.

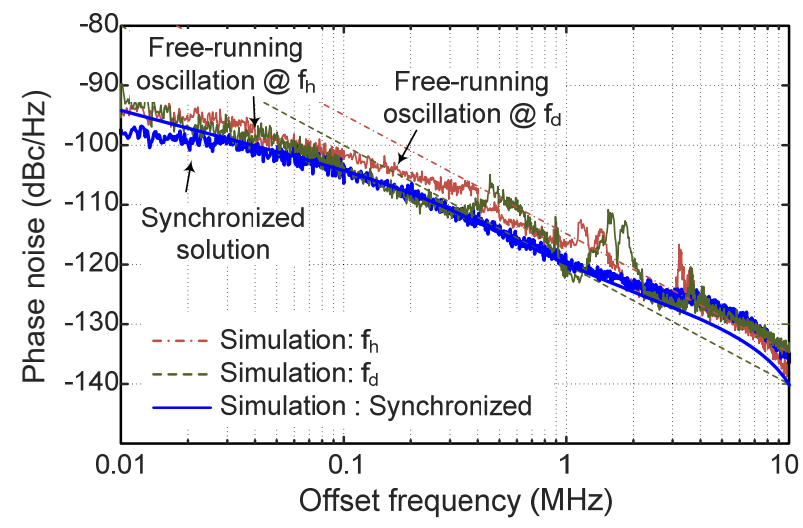

Fig. 14. Measured and simulated phase-noise spectra of the two individual periodic oscillations and the phase-locked solution. 


\section{CONCLUSION}

An insightful stability and bifurcation analysis of freerunning oscillators loaded with multi-resonant networks has been presented. It includes the cases of distinct periodic modes and concurrent dual-frequency oscillations, in a doubly autonomous quasi-periodic regime. Initially, the investigation has been illustrated through its application to a doublyresonance oscillator, based on an active device described with a polynomial model. This has enabled an exhaustive determination of all the coexisting steady-state solutions and, as a result, an overall understanding of the circuit behavior. Then, a practical oscillator based on a pair of cross-coupled transistors has been considered. Detailed stability and bifurcation analyses of $d c$, periodic and quasi-periodic solutions have been carried out. A new methodology for the stability analysis of doublyautonomous quasi-periodic solutions, obtained with harmonic balance, has been developed. The study provides insight into the various mechanisms leading to the generation of the periodic and quasi-periodic modes. It provides criteria to prevent the coexistence of stable periodic modes and to isolate a stable quasi-periodic solution from the periodic ones.

\section{REFERENCES}

[1] V. Rizzoli and A. Neri, "State of the art and present trends in nonlinear microwave CAD techniques," IEEE Trans. Microw. Theory Techn., vol. 36, no. 2, pp. 343-356, Feb., 1988.

[2] R. Quéré, E. Ngoya, M. Camiade, A. Suarez, M. Hessane and J. Obregon, "Large signal design of broadband monolithic microwave frequency dividers and phase-locked oscillators," IEEE Trans. Microw. Theory Techn., vol. 41, no. 11, pp. 1928-1938, Nov., 1993.

[3] S. Jeon, A. Suárez, R. Rutledge, "Global stability analysis and stabilization of a Class-E/F amplifier with a distributed active transformer," IEEE Trans. Microw. Theory Techn., vol. 53, no. 12, Dec. 2005, pp. 3712-3722.

[4] A. Suarez, J. Morales, R. Quéré, "Synchronization analysis of autonomous microwave circuits using new global-stability analysis tools," IEEE Trans. Microw. Theory Techn., vol. 46, no. 5, pp. 494-504, May 1998.

[5] K. Kurokawa, “An analysis of Rucker's multidevice symmetrical oscillator," IEEE Trans. Microw. Theory Techn., vol. 18, no. 5, pp. $967-$ 9, May 1970.

[6] R. G. Freitag, "A unified analysis of MMIC power amplifier stability," 1992 IEEE MTT-S Int. Microw. Symp. (IMS), Alburquerque, USA, 1992 pp. 297-300.

[7] F. Ramírez, M. Pontón, S. Sancho and A. Suárez, "Stability analysis of oscillation modes in quadruple-push and Rucker's oscillators," IEEE Trans. Microw. Theory Techn., vol. 56, no. 11, pp. 2648-61, Nov. 2008.

[8] M. Pontón, A. Suárez, S. Kenney, "Analysis and design of Rotary Travelling Wave Oscillators using Nonlinear Transmission Lines," IEEE Trans. Microw. Theory Techn., vol. 61, no. 1, pp. 4730-4744, Jan, 2014.

[9] J. de Cos, A. Suárez, and F. Ramírez, "Analysis of oscillation modes in free-running ring oscillators,” IEEE Trans. Microw. Theory Techn., vol. 60 , no. 10 , pp. $3137-3150$, Oct. 2012.

[10] A. Ballato, "Piezoelectric Resonators," in B. Parzen, Design of Crystal and Other Harmonic Oscillators, pp. 66-122, John Wiley and Sons, 1983.

[11] Z. Chengjie, et al., "Dual-Mode Resonator and Switchless Reconfigurable Oscillator Based on Piezoelectric AIN MEMS Technology," IEEE Trans. on Electron Devices, vol.58, no.10, pp .3599-3603, Oct. 2011.

[12] A. Goel and H. Hashemi, "Frequency Switching in Dual-Resonance Oscillators," IEEE Journal of Solid-State Circuits, vol. 42, no. 3, pp. 571582, March, 2007.

[13] J. S. Schaffner, "Simultaneous oscillations in oscillators", Transactions of the IRE, vol.CT-1, no.2, pp.2-8, June 1954.

[14] A. Grebennikov, "Stability analysis of wideband linear bipolar varactorcontrolled oscillator with two-coupled resonant circuits," International Journal of Numerical Modelling Electronic Networks Devices and Fields, Jun. 1999.
[15] I. Bruyland, "Simultanous oscillations at two frequencies in RLC circuits," Proc. IEEE, vol. 56, no. 5, pp. 895-903, Jan 1958.

[16] A. Bavisi et al. "A simultaneous asynchronous oscillator for simultaneous multi-band radio communication on a multi-layer liquid crystal polymer subtrate," IEEE Proc. Radiowireless Symposium, Jan. 2006, pp. 431-434.

[17] U. Rohde, A. Poddar, "Concurrent, reconfigurable and adaptable oscillators for multiband multimode communication systems," 37th European Microwave Conference, 2007, pp. 1050-1053.

[18] A. Goel and H. Hashemi, "Injection Locking in Concurrent DualFrequency Oscillators," IEEE Trans. Microw. Theory Techn., vol. 56, no. 8, pp. 1834-1845, Aug. 2008.

[19] A. Goel and H. Hashemi, "Phase noise in a synchronized concurrent dualfrequency oscillator," in Custom Integrated Circ. Conf., 2009, pp. 483486.

[20] J. Guckenheimer and P. Holmes, Nonlinear Oscillations, Dynamical Systems and Bifurcations of Vector Fields. New York: Springer-Verlag, 1990.

[21] S. Wiggins, Introduction to Applied Nonlinear Dynamical Systems and Chaos. New York: Springer-Verlag, 1990.

[22] T.S. Parker, L.O. Chua, Practical Numerical Algorithms for Chaotic Systems, Springer-Verlag, New York, 1989.

[23] F. Ramírez, A. Suárez, S. Sancho, "Analysis of oscillation modes in freerunning oscillators,” 2016 IEEE MTT-S Int. Microw. Symp. (IMS), San Francisco, CA, USA, May, 2016.

[24] A. Suárez, Analysis and Design of Autonomous Microwave Circuits, IEEE-Wiley, Hoboken, (NJ) Jan. 2009.

[25] A. Suárez, R. Quéré, Stability Analysis of Nonlinear Microwave Circuits. Artech House, Norwood (Ma), Jan. 2003.

[26] A. Gelb, W. Vander Velde, Multiple-input describing functions and nonlinear system design, McGraw-Hill, 1968.

[27] Ionita, M., Cordeau, D., Paillot, J. M., Bachir, S. and Iordache, M., "A CAD tool for an array of differential oscillators coupled through a broadband network," Int. J. RF and Microwave Comp. Aid. Eng., vol. 23, pp. 178-187, Aug., 2012.

[28] I. Dumitrescu, S. Bachir, D. Cordeau, J.-M. Paillot, M. Iordache, "Modeling and characterization of oscillator circuits by Van der Pol model using parameter estimation," J. Circuit Syst. Comp., vol. 21, no. 5, 2012.

[29] J. de Cos, A. Suárez, J. A. García, "Hysteresis and oscillation in high efficiency power amplifiers," IEEE Trans. Microw. Theory Techn., vol. 63, no. 12, pp., Dec. 2015.

[30] J. Jugo, J. Portilla, A. Anakabe, A. Suárez, and J. M. Collantes, "Closedloop stability analysis of microwave amplifiers," IEE Electronics Letters, vol. 37 , no. 4, pp. 226-228, Feb. 2001.

[31] N. Ayllon, J. M. Collantes, A. Anakabe, I. Lizarraga, S. Soubercaze-Pun, S. Forestier, "Systematic approach to the stabilization of multitransistor circuits", IEEE Trans. Microw. Theory Techn., vol. 59, no. 8, pp. 20732082, Aug. 2011

[32] F. Bonani and M. Gilli, "Analysis of stability and bifurcations of limit cycles in Chua's circuit through the harmonic-balance approach," IEEE Trans. Circuits Syst. I, Fundam. Theory Appl. (1993-2003), vol. 46, no. 8, pp. 881-890, Aug., 1999.

[33] K. Kurokawa, "Some basic characteristics of broadband negative resistance oscillators,” Bell Syst. Tech. J., vol. 48, pp. 1937-55, Jul. 1969.

[34] F. Ramírez, E. de Cos, A. Suárez, "Nonlinear analysis tools for the optimized design of harmonic-injection frequency dividers", IEEE Trans. Microw. Theory Techn., vol. 51, no. 6, pp. 1752-1762, Jun. 2003.

[35] M. M. Green and R. C. Melville, "Sufficient conditions for finding multiple operating points of DC circuits using continuation methods," 1995 IEEE Int. Symp. Circuits Syst. ISCAS, Seattle, WA, 1995, pp. 117-120 vol.1.

[36] A. Suárez, J. Morales, R. Quéré, "Synchronization analysis of autonomous microwave circuits using new global stability analysis tools," IEEE Trans. Microw. Theory Techn., vol. 46, no. 5, May 1998, pp. 494504.

[37] F. Ramírez, M. Pontón, S. Sancho, A. Suárez, "Phase-Noise Analysis of Injection-Locked Oscillators and Analog Frequency Dividers", IEEE Trans. Microw. Theory Techn., vol. 56, no.2, pp. 393-407, 2008.

Franco Ramírez was born in Potosí, Bolivia. He obtained a degree in electronic systems engineering degree from the Military School of Engineering (EMI) in La Paz, Bolivia, in 2000 and the Ph.D. degree in communications engineering from the University of Cantabria, Santander, Spain in 2005. From 
1999 to 2000 he worked for Ericsson de Bolivia Telecomunicaciones, where he was involved in several projects related with GSM and TDMA technologies. At present he is an Associate Professor at the Communications Engineering Department of the University of Cantabria. His research interests include phase noise, stability and the development of nonlinear techniques for the analysis and design of autonomous microwave circuits.

Sergio Sancho was born in Santurce, Spain, in 1973. In 1997 received the degree in Physics from Basque Country University. In 1998 he joined the Communications Engineering Department of the University of Cantabria, Spain, where he received the Ph.D. degree in Electronic Engineering in February 2002. At present, he works at the University of Cantabria, as an Associate Professor of its Communications Engineering Department. His research interests include the nonlinear analysis of microwave autonomous circuits and frequency synthesizers, including stochastic and phase-noise analysis.

Almudena Suárez (M'96-SM'01-F'12) was born in Santander, Spain. She received the Electronic Physics and Ph.D. degrees from the University of Cantabria, Santander, Spain, in 1987 and 1992, respectively, and the Ph.D. degree in electronics from the University of Limoges, Limoges, France, in 1993.

She is currently a Full Professor with the Communications Engineering Department, University of Cantabria. She co-authored Stability Analysis of Nonlinear Microwave Circuits (Artech House, 2003) and authored Analysis and Design of Autonomous Microwave Circuits (IEEE-Wiley, 2009).

Prof. Suárez is a member of the Technical Committees of the IEEE Microwave Theory and Techniques Society (IEEE MTT-S) International Microwave Symposium (IMS) and the European Microwave Conference. She was an IEEE Distinguished Microwave Lecturer from 2006 to 2008. She is a member of the Board of Directors of the European Microwave Association. She is the Editorin-Chief of the International Journal of Microwave and Wireless Technologies (Cambridge University Press). She was the co-chair of IEEE Topical Conference on RF Power Amplifiers (PAWR) in 2014 and 2015. 